\title{
Tailoring the spatiotemporal structure of biphoton entanglement in type-I parametric down-conversion
}

\author{
L. Caspani, E. Brambilla, and A. Gatti \\ CNR-CNISM, Dipartimento di Fisica e Matematica, Università dell'Insubria, via Valleggio 11, I-22100 Como, Italy
}

(Received 29 July 2009; published 8 March 2010)

\begin{abstract}
We investigate the spatiotemporal structure of the biphoton entangled state produced by parametric downconversion (PDC) at the output face of the nonlinear crystal. We analyze the geometry of biphoton correlation for different gain regimes (from ultralow to high), different crystal lengths, and different tuning angles of the crystal. While for collinear or quasicollinear phase matching a X-shaped geometry, nonfactorizable in space and time, dominates, in the highly noncollinear conditions we observe a remarkable transition to a factorizable geometry. We show that the geometry of spatiotemporal correlation is a consequence of the angle-frequency relationship imposed by phase matching and that the fully spatiotemporal analysis provides a key to control the spatiotemporal properties of the PDC entangled state and in particular to access a biphoton localization in time and space in the femtosecond and micrometer range, respectively.
\end{abstract}

DOI: 10.1103/PhysRevA.81.033808

PACS number(s): 42.65.Lm, 42.65.Sf, 42.50.Ar, 42.50.Dv

\section{INTRODUCTION}

The entangled photon pairs (biphotons) produced in the parametric down-conversion (PDC) process are the key element for several quantum information and communication schemes. According to the application considered, different properties of the entangled photons (e.g., the spectral bandwidth) are enhanced. For example, recently proposed protocols for long-distance quantum communication based on photonatom interactions requires narrow-band biphotons [1,2] with linewidth less than the atomic linewidth $(\sim \mathrm{MHz}[3,4])$, whereas ultrabroadband biphotons $(\sim \mathrm{GHz})$ are required for high-axial-resolution quantum optical coherence tomography $[5,6]$ or for clock synchronization protocols [7,8]. On the other hand, broadband photons in the spatial domain are required by applications in quantum imaging [9] or quantum lithography [10], where the spatial localization of biphotons sets the limit of spatial resolution.

Different methods have been proposed in recent years to generate broadband biphotons and more in general to tailor their spectral properties. Some of them exploit the properties of the nonlinear medium by choosing a very thin crystal [11] or by using chirped quasi-phase-matched nonlinear gratings [12-15]. In other works the spectral properties of biphotons are controlled thanks to pump engineering by choosing appropriately the pump wavelength [16,17], acting on its spatial properties and exploiting the noncollinear phasematching configuration [18-20], or, more recently, by means of a tilted pump pulse [21].

In a recent article [22] we demonstrated that the structure of the PDC entanglement is not factorizable with respect to the spatial and temporal variables, which means that a complete description of the state can be done only in a nonseparable framework. In particular we showed that the biphoton amplitude at the output face of the crystal (near-field) has an X-shaped structure that we referred to as X-entanglement. The nonfactorizability of the PDC state opens the possibility of tailoring the temporal bandwidth of the biphotons by manipulating their spatial degrees of freedom. In particular by resolving the near-field position of twin photons it is possible to access an ultrabroadband source of entangled photons, with a temporal localization of a few femtoseconds. This extreme localization is an intrinsic property of biphotons produced in the PDC process: We do not need a particular nonlinear medium or particular properties of the pump pulse but only the correct detection technique that is able to resolve the $\mathrm{X}$ structure.

In Ref. [22] we focused on the ultralow gain regime of PDC, where single photon pairs are produced and detected, and on the collinear phase-matching case. In this work we extend our analysis to the high gain regime of PDC and to different phase matching conditions; we also study the dependence of the spatiotemporal structure of the correlation on the crystal length. As in the previous work we focus our attention to type I PDC process. A detailed study of the biphoton amplitude in type II PDC, also showing a nonseparable spatiotemporal structure, is given in Ref. [23].

We show that in the collinear case, in the high gain regime where macroscopic signal and idler fields are produced, their spatiotemporal correlation maintains the same X-geometry and nonfactorizable character with respect to space and time as the biphoton amplitude in the low gain regime. By studying the dependence of the phenomenon on the crystal length, we show how an $\mathrm{X}$ structure emerges gradually by increasing the length of the nonlinear medium, and we elucidate its close relationship with the phase-matching mechanism that is at the base of PDC. Differing from other detection schemes, where typically the temporal localization of biphotons broadens by increasing the crystal length, we show that by resolving the near-field position of biphotons, their temporal localization is independent of the crystal length and therefore an ultranarrow temporal localization can be achieved for arbitrarily long crystals.

Since one of the key elements to completely understand the spatiotemporal structure of biphotons is its close relationship with the phase-matching mechanism, we analyze the effects of different phase-matching conditions on the $\mathrm{X}$ structure. In particular by changing the tuning angle between the pump propagation direction and the principal axis of the nonlinear crystal, we observe an interesting transition from 
the nonseparable structure to a separable one. A remarkable characteristic of the factorizable correlation that emerges in these conditions is that a femtosecond temporal localization of biphotons is still present independent of the type of the spatial measurement. At the same time, an ultranarrow submicron spatial localization can be achieved independent of the temporal measurement, making this situation particularly attractive for applications in quantum imaging [9] and quantum lithography [10].

All these features can be clearly described by means of a fully spatiotemporal analysis. The nonfactorizability in space and time of the PDC state and its close relationship with the phase-matching mechanism are the key elements to completely access and control the properties of the entangled photon pairs.

The article is organized as follows. In Sec. II we describe the model that we adopt to describe the PDC process. We start from the low gain regime (Sec. II A). In Sec. II B we describe the similarities and the differences between the biphoton amplitude and the coherence function, which are the main quantities in the description of the PDC process. Section II C is devoted to the high gain regime. Section III will describe our results on the biphoton correlation in the case of collinear phase matching (Sec. III A), the effect of the crystal length (Sec. III B), and the transition from the X-shaped geometry, nonseparable in space and time, to a factorizable structure, obtained by changing the crystal tuning angle (Sec. III C).

\section{THE MODEL}

The model we adopt to describe the parametric downconversion process is similar to the one described in Refs. [9,24,25], the main difference being that we shall not make the narrow-band approximation. A coherent and quasimonochromatic pump field propagates along the $z$ axis in a $\chi^{(2)}$ nonlinear crystal of length $l_{c}$, cut for type I e-oo phase matching ("e" indicates the extraordinary polarized pump while "o" indicates the ordinary polarized signal field). We denote by $\hat{A}_{p}(\vec{x}, t, z)$ and $\hat{A}_{s}(\vec{x}, t, z)$ the envelope operators for the pump and signal fields, of central frequencies $\omega_{p}$ and $\omega_{s}=\omega_{p} / 2$, respectively:

$$
\hat{A}_{j}(\vec{x}, t, z)=\hat{E}_{j}^{(+)}(\vec{x}, t, z) e^{i \omega_{j} t} \quad(j=s, p),
$$

where $\hat{E}_{j}^{(+)}$(with dimensions of a photon annihilation operator) is the positive frequency part of the field operator and $\vec{x}=(x, y)$ represents the transverse coordinates, while $t$ is the time. We next pass to the Fourier domain:

$$
\hat{A}_{j}(\vec{q}, \Omega, z)=\int \frac{d^{2} \vec{x}}{2 \pi} \int \frac{d t}{\sqrt{2 \pi}} e^{-i \vec{q} \cdot \vec{x}+i \Omega t} \hat{A}_{j}(\vec{x}, t, z),
$$

where $\vec{q}$ represents the transverse component of the wave vector and $\Omega$ is the frequency offset from the carrier frequency $\omega_{j}$. In addition we extract the fast variation along $z$ due to the linear propagation inside the crystal:

$$
\hat{A}_{j}(\vec{q}, \Omega, z)=e^{i k_{j z}(\vec{q}, \Omega) z} \hat{a}_{j}(\vec{q}, \Omega, z),
$$

where $k_{j z}(\vec{q}, \Omega)=\sqrt{k_{j}^{2}(\vec{q}, \Omega)-q^{2}}$ is the projection, along the $z$ axis, of the wave vector of the $j$-th field. The corresponding wave number, that for an ordinary wave does not depend on $\vec{q}$, is $k_{j}(\vec{q}, \Omega)=n\left(\vec{q}, \omega_{j}+\Omega\right)\left(\omega_{j}+\Omega\right) / c_{0}$, where $n$ is the refractive index and $c_{0}$ the light speed in vacuum.

The fields $\hat{a}_{j}$ defined in Eq. (3) have thus a slow variation along the crystal, due only to the nonlinear interaction. In the following we assume that the pump beam is undepleted by the nonlinear interaction, i.e., $\hat{a}_{p}(\vec{q}, \Omega, z)=\hat{a}_{p}(\vec{q}, \Omega, 0)$. Moreover, assuming the pump is an intense coherent beam, its field operator can be replaced by the classical mean value: $\hat{a}_{p}(\vec{q}, \Omega, 0) \rightarrow \alpha_{p}(\vec{q}, \Omega)$. In this way the pump evolution along the crystal is simply determined by the linear propagation, $\mathcal{A}_{p}(\vec{q}, \Omega, z)=e^{i k_{p z}(\vec{q}, \Omega) z} \alpha_{p}(\vec{q}, \Omega)$.

The evolution of the signal field $\hat{a}$ due to the nonlinear interaction (we can, from now on, omit the subscript " $s$ " without creating confusion, since the pump field is represented by a $c$ number), is described by the following equation [24]:

$$
\begin{aligned}
\frac{\partial \hat{a}(\vec{q}, \Omega, z)}{\partial z}= & \frac{g}{l_{c}} \int \frac{d^{2} \vec{q}^{\prime}}{2 \pi} \int \frac{d \Omega^{\prime}}{\sqrt{2 \pi}} \bar{\alpha}_{p}\left(\vec{q}+\vec{q}^{\prime}, \Omega+\Omega^{\prime}\right) \\
& \times \hat{a}^{\dagger}\left(\vec{q}^{\prime}, \Omega^{\prime}, z\right) e^{-i \Delta\left(\vec{q}, \Omega, \vec{q}^{\prime}, \Omega^{\prime}\right) z},
\end{aligned}
$$

where $\bar{\alpha}_{p}$ represents the pump field (at the input face of the crystal) normalized to its peak value $\alpha_{p}$, and $g=\chi^{(2)} \alpha_{p} l_{c}$ is the dimensionless parametric gain, with $\chi^{(2)}$ being a parameter proportional to the second-order susceptibility of the medium. The phase mismatch function, defined as

$$
\begin{aligned}
& \Delta\left(\vec{q}, \Omega, \vec{q}^{\prime}, \Omega^{\prime}\right) \\
& \quad=k_{s z}(\vec{q}, \Omega)+k_{s z}\left(\vec{q}^{\prime}, \Omega^{\prime}\right)-k_{p z}\left(\vec{q}+\vec{q}^{\prime}, \Omega+\Omega^{\prime}\right),
\end{aligned}
$$

determines the efficiency of the down-conversion process, in which a pump photon of frequency $\omega_{p}+\Omega+\Omega^{\prime}$ with transverse wave vector $\vec{q}+\vec{q}^{\prime}$, splits into two signal photons of frequencies $\omega_{s}+\Omega$ and $\omega_{s}+\Omega^{\prime}$, with wave vectors $\vec{q}$ and $\vec{q}^{\prime}$, respectively.

\section{A. Low gain regime}

Equation (4) can be analytically solved for a generic gain only within the stationary and plane-wave pump approximation (PWPA). In the low gain regime $(g \ll 1)$ instead, a perturbative approach [26,27] allows us to solve Eq. (4) up to first order in $g$ (see Appendix A):

$$
\begin{aligned}
\hat{A}(\vec{q}, \Omega)= & e^{i k_{s z}(\vec{q}, \Omega) l_{c}}\left[\hat{A}_{\mathrm{in}}(\vec{q}, \Omega)+g \int \frac{d^{2} \vec{q}^{\prime} d \Omega^{\prime}}{(2 \pi)^{3 / 2}}\right. \\
& \times \bar{\alpha}_{p}\left(\vec{q}+\vec{q}^{\prime}, \Omega+\Omega^{\prime}\right) \hat{A}_{\mathrm{in}}^{\dagger}\left(\vec{q}^{\prime}, \Omega^{\prime}\right) e^{-i \frac{\Delta\left(\vec{q}, \Omega, \vec{q}^{\prime}, \Omega^{\prime}\right)}{2} l_{c}} \\
& \left.\times \operatorname{sinc}\left(\frac{\Delta\left(\vec{q}, \Omega, \vec{q}^{\prime}, \Omega^{\prime}\right)}{2} l_{c}\right)\right]
\end{aligned}
$$

where $\operatorname{sinc}(x) \equiv \sin (x) / x$ and, if not differently stated, the field $\hat{A}$ is evaluated at $z=l_{c}$ (near field), while we denote $\hat{A}_{\text {in }}$ the field at the input face of the crystal $(z=0)$.

The quantity of primary interest when dealing with the PDC entangled state is the biphoton cross correlation or biphoton amplitude:

$$
\psi\left(\vec{x}, t, \vec{x}^{\prime}, t^{\prime}\right)=\left\langle\hat{A}(\vec{x}, t) \hat{A}\left(\vec{x}^{\prime}, t^{\prime}\right)\right\rangle,
$$

which is at the heart of the photon-pair PDC entanglement. The "anomalous" propagator shown in Eq. (7) is, indeed, characteristic of processes where particles are created in pairs. As we shall show in the following, in the low gain regime its square modulus is proportional to the joint probability 
distribution $G^{(2)}\left(\vec{x}, t, \vec{x}^{\prime}, t^{\prime}\right)$ of finding two photons, one at position $\vec{x}$ at time $t$ and the other at position $\vec{x}^{\prime}$ at time $t^{\prime}$.

From Eq. (6) we can derive an expression for the biphoton amplitude in the Fourier domain:

$$
\begin{aligned}
\left\langle\hat{A}(\vec{q}, \Omega) \hat{A}\left(\vec{q}^{\prime}, \Omega^{\prime}\right)\right\rangle & \\
= & \frac{g}{(2 \pi)^{3 / 2}} \bar{\alpha}_{p}\left(\vec{q}+\vec{q}^{\prime}, \Omega+\Omega^{\prime}\right) e^{i\left[k_{s z}(\vec{q}, \Omega)+k_{s z}\left(\vec{q}^{\prime}, \Omega^{\prime}\right)\right] l_{c}} \\
& \times e^{-i \frac{\Delta\left(\vec{q}, \Omega, \vec{q}^{\prime}, \Omega^{\prime}\right)}{2} l_{c}} \operatorname{sinc}\left(\frac{\Delta\left(\vec{q}, \Omega, \vec{q}^{\prime}, \Omega^{\prime}\right)}{2} l_{c}\right) \\
= & \frac{g}{(2 \pi)^{3 / 2}} \overline{\mathcal{A}}_{p}\left(\vec{q}+\vec{q}^{\prime}, \Omega+\Omega^{\prime}, l_{c}\right) \\
& \times e^{i \frac{\Delta\left(\vec{q}, \Omega, \vec{q}^{\prime}, \Omega^{\prime}\right)}{2} l_{c}} \operatorname{sinc}\left(\frac{\Delta\left(\vec{q}, \Omega, \vec{q}^{\prime}, \Omega^{\prime}\right)}{2} l_{c}\right),
\end{aligned}
$$

where $\overline{\mathcal{A}}_{p}\left(\vec{q}, \Omega, l_{c}\right)=e^{i k_{p z}(\vec{q}, \Omega) l_{c}} \bar{\alpha}_{p}(\vec{q}, \Omega)$ is the Fourier transform of the normalized pump envelope at the output face of the crystal, and we exploited the standard commutation relations at equal $z$ :

$$
\left[\hat{A}_{\mathrm{in}}(\vec{q}, \Omega), \hat{A}_{\mathrm{in}}^{\dagger}\left(\vec{q}^{\prime}, \Omega^{\prime}\right)\right]=\delta\left(\vec{q}-\vec{q}^{\prime}\right) \delta\left(\Omega-\Omega^{\prime}\right),
$$

and the identities $\left\langle\hat{A}_{\text {in }} \hat{A}_{\text {in }}\right\rangle=\left\langle\hat{A}_{\text {in }}^{\dagger} \hat{A}_{\text {in }}\right\rangle=\left\langle\hat{A}_{\text {in }}^{\dagger} \hat{A}_{\text {in }}^{\dagger}\right\rangle=0$, deriving from the fact that the signal field at input face of the crystal is in the vacuum state.

We are interested in calculating the biphoton amplitude in the direct domain, that is, the inverse Fourier transform of Eq. (8):

$$
\begin{aligned}
\psi\left(\vec{x}, t, \vec{x}^{\prime}, t^{\prime}\right)= & \int \frac{d^{2} \vec{q} d \Omega}{(2 \pi)^{3 / 2}} \int \frac{d^{2} \vec{q}^{\prime} d \Omega^{\prime}}{(2 \pi)^{3 / 2}}\left\langle\hat{A}(\vec{q}, \Omega) \hat{A}\left(\vec{q}^{\prime}, \Omega^{\prime}\right)\right\rangle \\
& \times e^{i(\vec{q} \cdot \vec{x}-\Omega t)} e^{i\left(\vec{q}^{\prime} \cdot \vec{x}^{\prime}-\Omega^{\prime} t^{\prime}\right)}
\end{aligned}
$$

In order to simplify the analysis, we shall consider a limit of a pump beam quasistationary and quasihomogeneous. We introduce the following change of variables:

$$
\left\{\begin{array}{l}
\left(\vec{q}_{p}, \Omega_{p}\right)=\left(\vec{q}+\vec{q}^{\prime}, \Omega+\Omega^{\prime}\right), \\
\left(\vec{q}_{s}, \Omega_{s}\right)=\left(\frac{\vec{q}-\vec{q}^{\prime}}{2}, \frac{\Omega-\Omega^{\prime}}{2}\right) .
\end{array}\right.
$$

The phase mismatch function takes thus the form

$$
\begin{aligned}
\Delta\left(\vec{q}_{s}, \Omega_{s}, \vec{q}_{p}, \Omega_{p}\right)= & k_{s z}\left(\vec{q}_{s}+\frac{\vec{q}_{p}}{2}, \Omega_{s}+\frac{\Omega_{p}}{2}\right) \\
& +k_{s z}\left(-\vec{q}_{s}+\frac{\vec{q}_{p}}{2},-\Omega_{s}+\frac{\Omega_{p}}{2}\right) \\
& -k_{p z}\left(\vec{q}_{p}, \Omega_{p}\right) .
\end{aligned}
$$

If we suppose now that the variation of $\Delta$ with respect to the pump variables, $\vec{q}_{p}$ and $\Omega_{p}$, is very slow compared to the scale over which the pump $\overline{\mathcal{A}}_{p}$ dies out, we can approximate $\Delta\left(\vec{q}_{s}, \Omega_{s}, \vec{q}_{p}, \Omega_{p}\right) \sim \Delta\left(\vec{q}_{s}, \Omega_{s}, 0,0\right)$. We shall refer to this approximation as the nearly plane-wave and monochromatic pump approximation (NPWPA). The NPWPA is not too difficult to achieve: as will be explained in detail in Appendix B, it holds when the pump pulse duration and its transverse size are large enough compared to the characteristic temporal and lateral walk-off experienced by the signal and pump photons when crossing the crystal [a few picoseconds and hundreds of microns, respectively, for a 4-mm beta barium borate (BBO) crystal].
Under these circumstances, the field correlation in the Fourier domain factorizes into the product of two functions, one that depends on the pump variables $\left(\vec{q}_{p}, \Omega_{p}\right)$ and the other on the "mean" signal variables $\left(\vec{q}_{s}, \Omega_{s}\right)$ :

$$
\begin{aligned}
\psi\left(\vec{q}_{s}, \Omega_{s}, \vec{q}_{p}, \Omega_{p}\right)= & \overline{\mathcal{A}}_{p}\left(\vec{q}_{p}, \Omega_{p}, l_{c}\right)\left[\frac{g}{(2 \pi)^{3 / 2}} e^{i \frac{\Delta\left(\vec{q}_{s}, \Omega_{s}, 0,0\right)}{2} l_{c}}\right. \\
& \left.\times \operatorname{sinc}\left(\frac{\Delta\left(\vec{q}_{s}, \Omega_{s}, 0,0\right)}{2} l_{c}\right)\right] .
\end{aligned}
$$

The field correlation function in the direct domain takes thus the factorized form:

$$
\begin{aligned}
\psi\left(\vec{x}, t, \vec{x}^{\prime}, t^{\prime}\right)= & \int \frac{d^{2} \vec{q}_{p} d \Omega_{p} d^{2} \vec{q}_{s} d \Omega_{s}}{(2 \pi)^{3}} \\
& \times e^{i\left[\vec{q}_{p} \cdot\left(\vec{x}+\vec{x}^{\prime}\right) / 2-\Omega_{p}\left(t+t^{\prime}\right) / 2+\vec{q}_{s} \cdot\left(\vec{x}-\vec{x}^{\prime}\right)-\Omega_{s}\left(t-t^{\prime}\right)\right]} \\
& \times \psi\left(\vec{q}_{s}, \Omega_{s}, \vec{q}_{p}, \Omega_{p}\right) \\
= & \overline{\mathcal{A}}_{p}\left(\frac{\vec{x}+\vec{x}^{\prime}}{2}, \frac{t+t^{\prime}}{2}, l_{c}\right) \psi_{\mathrm{pw}}\left(\vec{x}-\vec{x}^{\prime}, t-t^{\prime}\right),
\end{aligned}
$$

where

$$
\begin{gathered}
\psi_{\mathrm{pw}}\left(\vec{x}-\vec{x}^{\prime}, t-t^{\prime}\right)=\int \frac{d^{2} \vec{q} d \Omega}{(2 \pi)^{3}} e^{i \vec{q} \cdot\left(\vec{x}-\vec{x}^{\prime}\right)-i \Omega\left(t-t^{\prime}\right)} \mathcal{V}(\vec{q}, \Omega), \\
\mathcal{V}(\vec{q}, \Omega)=g e^{i \frac{\Delta \mathrm{pw}(\vec{q}, \Omega)}{2} l_{c}} \operatorname{sinc}\left(\frac{\Delta_{\mathrm{pw}}(\vec{q}, \Omega)}{2} l_{c}\right), \\
\Delta_{\mathrm{pw}}(\vec{q}, \Omega)=k_{s z}(\vec{q}, \Omega)+k_{s z}(-\vec{q},-\Omega)-k_{p}(0,0),
\end{gathered}
$$

is the plane wave pump (PWP) result for the biphoton amplitude. It means that, in the low gain regime, when the NPWPA holds, the pump profile, as a function of the mean variables, acts as a slow modulation of the PWP result, which is a function only of the relative coordinates $\vec{\xi}=\vec{x}-\vec{x}^{\prime}$ and $\tau=$ $t-t^{\prime}$. Therefore by adopting the NPWPA, the calculation of the biphoton amplitude reduces to the evaluation of the second term of Eq. (14), which is the plane-wave and monochromatic pump result.

As shown in more details in Ref. [22], if we consider in addition to the low gain limit, the paraxial and quadratic dispersion approximation for the phase mismatch function it is possible to develop a toy model that leads to an analytic expression for $\psi_{\mathrm{pw}}$ (without this last approximation $\psi_{\mathrm{pw}}$ need to be numerically evaluated) that evidences its X structure, not separable in space and time. It is indeed possible to show that, within these approximations, the biphoton amplitude assumes constant values on the rotational hyperboloid family [22]:

$$
q_{0}^{2} \xi^{2}-\Omega_{0}^{2} \tau^{2}=\text { constant }
$$

where $\xi=|\vec{\xi}|, q_{0}=\sqrt{k_{s} / l_{c}}, \Omega_{0}=\sqrt{1 / k_{s}^{\prime \prime} l_{c}}$, and we used the short-hand notation $k_{s}=k_{s}(0), k_{p}=k_{p}(0,0), \quad k_{s}^{\prime \prime}=$ $d^{2} k_{s} /\left.d \Omega^{2}\right|_{0}$ (we remind that since the signal is an ordinary wave its wave number depends only on $\Omega$, while the pump wave number depends on both $\vec{q}$ and $\Omega$ ). The analytic expression found in Ref. [22] demonstrates the hyperbolic geometry of the biphoton amplitude; however, it diverges when approaching the asymptotes of the $\mathrm{X}$ structure, defined by

$$
q_{0}^{2} \xi^{2}-\Omega_{0}^{2} \tau^{2}=0 \Rightarrow \pm \xi=\frac{\Omega_{0}}{q_{0}} \tau .
$$


Because of this divergence we cannot obtain quantitative results from the analytic expression. We shall in fact in the following evaluate the biphoton amplitude (15) numerically.

\section{B. Biphoton amplitude and coherence function}

Before extending the study of the biphoton amplitude to different gain regimes, we open a brief parenthesis about the relationship between the biphoton amplitude and the classical coherence function

$$
G^{(1)}\left(\vec{x}, t, \vec{x}^{\prime}, t^{\prime}\right) \equiv\left\langle\hat{A}^{\dagger}(\vec{x}, t) \hat{A}\left(\vec{x}^{\prime}, t^{\prime}\right)\right\rangle .
$$

This is the other quantity of interest when dealing with Gaussian processes, such as PDC. Thanks to the quantum version of the Gaussian moment-factoring theorem [28], it is indeed possible to calculate all the expectation values and the correlation functions of interest only by means of the second-order moments of field operators, i.e., $\langle\hat{A} \hat{A}\rangle$ and $\left\langle\hat{A}^{\dagger} \hat{A}\right\rangle$.

Following the same reasoning as for the biphoton amplitude, we find that, in the low gain regime and in the NPWPA, the coherence function takes the form:

$$
\begin{aligned}
G^{(1)}\left(\vec{x}, t, \vec{x}^{\prime}, t^{\prime}\right)= & \left|\overline{\mathcal{A}}_{p}\left(\frac{\vec{x}+\vec{x}^{\prime}}{2}, \frac{t+t^{\prime}}{2}, l_{c}\right)\right|^{2} \\
& \times G_{\mathrm{pw}}^{(1)}\left(\vec{x}-\vec{x}^{\prime}, t-t^{\prime}\right),
\end{aligned}
$$

where

$G_{\mathrm{pw}}^{(1)}\left(\vec{x}-\vec{x}^{\prime}, t-t^{\prime}\right)=\int \frac{d^{2} \vec{q} d \Omega}{(2 \pi)^{3}} e^{-i \vec{q} \cdot\left(\vec{x}-\vec{x}^{\prime}\right)} e^{i \Omega\left(t-t^{\prime}\right)}|\mathcal{V}(\vec{q}, \Omega)|^{2}$

is the PWP result for the coherence function of the downconverted field, with $\mathcal{V}(\vec{q}, \Omega)$ defined in Eq. (15b). We note that since $\mathcal{V}(\vec{q}, \Omega)$ is proportional to the gain $g$, the biphoton amplitude scales as $g$ [see Eq. (15a)] while the coherence function scales as $g^{2}$.

The joint probability distribution $G^{(2)}\left(\vec{x}, t, \vec{x}^{\prime}, t^{\prime}\right)$ of finding two photons, one at position $\vec{x}$ at time $t$ and the other at position $\vec{x}^{\prime}$ at time $t^{\prime}$, is proportional to the second-order intensity correlation function [29]:

$G^{(2)}\left(\vec{x}, t, \vec{x}^{\prime}, t^{\prime}\right)=\left\langle\hat{A}^{\dagger}(\vec{x}, t) \hat{A}^{\dagger}\left(\vec{x}^{\prime}, t^{\prime}\right) \hat{A}\left(\vec{x}^{\prime}, t^{\prime}\right) \hat{A}(\vec{x}, t)\right\rangle$.

By using Eqs. (2) and (6) together with the commutation relations (9), after some long but not difficult calculations we obtain:

$$
\begin{aligned}
G^{(2)}\left(\vec{x}, t, \vec{x}^{\prime}, t^{\prime}\right)= & \left|\psi\left(\vec{x}, t, \vec{x}^{\prime}, t^{\prime}\right)\right|^{2}+\left|G^{(1)}\left(\vec{x}, t, \vec{x}^{\prime}, t^{\prime}\right)\right|^{2} \\
& +\langle I(\vec{x}, t)\rangle\left\langle I\left(\vec{x}^{\prime}, t^{\prime}\right)\right\rangle
\end{aligned}
$$

where $\langle I(\vec{x}, t)\rangle=G^{(1)}(\vec{x}, t, \vec{x}, t)$ is the mean intensity distribution of the field at position $\vec{x}$ at time $t$.

Since the first term is proportional to $g^{2}$, while the last two terms are proportional to $g^{4}$, in the low gain regime $(g \ll 1)$ we have:

$$
G^{(2)}\left(\vec{x}, t, \vec{x}^{\prime}, t^{\prime}\right)=\left|\psi\left(\vec{x}, t, \vec{x}^{\prime}, t^{\prime}\right)\right|^{2}+O\left(g^{4}\right),
$$

and the square modulus of $\psi$ is thus proportional to the joint probability of detecting one photon at position $\vec{x}$ and time $t$ and the other at position $\vec{x}^{\prime}$ and time $t^{\prime}$. It has been recently demonstrated that also the coherence volume, described by
$G^{(1)}\left(\vec{x}, t, \vec{x}^{\prime}, t^{\prime}\right)$, of the field generated in type I parametric down-conversion, is not separable in space and time and has an X-shaped structure [30,31].

We recall that the coherence function describes the coherence properties of the individual signal or idler field, and in the high gain regime it accounts for the autocorrelation of the signal (idler) field with itself, whereas the biphoton amplitude describes the cross correlation of the entangled photons (say signal and idler photons) produced in the elementary process of photon pairs generation that is at the root of PDC.

Supposing we are able to separate a signal photon from its conjugate twin, we can enhance the difference between these two quantities. For example, by assuming that the pump is a broad-enough pulse (e.g., a broad Gaussian pulse), Eq. (8) tells us that only photons that propagates with symmetric $\vec{q}$ and $-\vec{q}$ are correlated. We can then separate the down-converted field with respect to the propagation direction, that is, we define:

$$
\begin{aligned}
& \hat{A}_{1}(\vec{q}, \Omega)=\left\{\begin{array}{ll}
\hat{A}(\vec{q}, \Omega) & \text { for } q_{x} \geqslant 0 \\
\text { vacuum } & \text { otherwise, }
\end{array}\right. \text { and } \\
& \hat{A}_{2}(\vec{q}, \Omega)= \begin{cases}\hat{A}(\vec{q}, \Omega) & \text { for } q_{x}<0 \\
\text { vacuum } & \text { otherwise. }\end{cases}
\end{aligned}
$$

In this way, each photon in $\hat{A}_{1}$ has its twin photon in $\hat{A}_{2}$, and the biphoton amplitude takes the form

$$
\psi\left(\vec{x}, t, \vec{x}^{\prime}, t^{\prime}\right)=\left\langle\hat{A}_{1}(\vec{x}, t) \hat{A}_{2}\left(\vec{x}^{\prime}, t^{\prime}\right)\right\rangle
$$

Note that, by using Eq. (8), $\left\langle\hat{A}_{1}(\vec{x}, t) \hat{A}_{1}\left(\vec{x}^{\prime}, t^{\prime}\right)\right\rangle=0=$ $\left\langle\hat{A}_{2}(\vec{x}, t) \hat{A}_{2}\left(\vec{x}^{\prime}, t^{\prime}\right)\right\rangle$.

For the classical coherence function, we have:

$$
G_{i i}^{(1)}\left(\vec{x}, t, \vec{x}^{\prime}, t^{\prime}\right)=\left\langle\hat{A}_{i}^{\dagger}(\vec{x}, t) \hat{A}_{i}\left(\vec{x}^{\prime}, t^{\prime}\right)\right\rangle, \quad i=1,2,
$$

while $\left\langle\hat{A}_{1}^{\dagger}(\vec{x}, t) \hat{A}_{2}\left(\vec{x}^{\prime}, t^{\prime}\right)\right\rangle=0$, identically.

The similarity between the $\psi$ and $G^{(1)}$ structures (both are $\mathrm{X}$ shaped, not separable in space and time) is the direct consequence of the cascading effect, where the photons already generated seed a new generation process. The primary process is the generation of a couple of twin photons, with the crosscorrelation properties previously discussed and described by $\psi$. Because of cascading, the shape of the cross correlation in space-time is then transferred also to the autocorrelation function, described by $G^{(1)}$, in a second-order process. Indeed we have that $\psi$ is proportional to $g$, while $G^{(1)}$ scales as $g^{2}$.

\section{Generic gain regime}

When the gain parameter is on the order of unity, $g=O(1)$, a perturbative expansion of the solution of the propagation equation of the form (6) is no more possible. In this case, analytic results can be obtained only by using directly the plane-wave and monochromatic pump limit $\bar{\alpha}_{p}(\vec{q}, \Omega) \rightarrow$ $(2 \pi)^{3 / 2} \delta(\vec{q}) \delta(\Omega)$.

The solution in this case can be expressed by means of the usual input-output relations [24,25,32]:

$\hat{A}(\vec{q}, \Omega)=U(\vec{q}, \Omega) \hat{A}_{\text {in }}(\vec{q}, \Omega)+V(\vec{q}, \Omega) \hat{A}_{\text {in }}^{\dagger}(-\vec{q},-\Omega)$, 
where

$$
\begin{aligned}
& U(\vec{q}, \Omega)=e^{i \frac{k p l_{c}}{2}} e^{i \varphi(\vec{q}, \Omega)}\{\cosh [\Gamma(\vec{q}, \Omega)] \\
& \left.+i \frac{\Delta_{\mathrm{pw}}(\vec{q}, \Omega) l_{c}}{2 \Gamma(\vec{q}, \Omega)} \sinh [\Gamma(\vec{q}, \Omega)]\right\}, \\
& V(\vec{q}, \Omega)=e^{i \frac{k p l_{c}}{2}} e^{i \varphi(\vec{q}, \Omega)} \frac{g}{\Gamma(\vec{q}, \Omega)} \sinh [\Gamma(\vec{q}, \Omega)], \\
& \varphi(\vec{q}, \Omega)=\frac{k_{s z}(\vec{q}, \Omega)-k_{s z}(-\vec{q},-\Omega)}{2} l_{c}, \\
& \Gamma(\vec{q}, \Omega)=\sqrt{g^{2}-\frac{\Delta_{\mathrm{pw}}^{2}(\vec{q}, \Omega) l_{c}^{2}}{4}} .
\end{aligned}
$$

We note that the functions $U(\vec{q}, \Omega)$ and $V(\vec{q}, \Omega)$ satisfy the conditions:

$$
\begin{aligned}
|U(\vec{q}, \Omega)|^{2}-|V(\vec{q}, \Omega)|^{2} & =1, \\
U(\vec{q}, \Omega) V(-\vec{q},-\Omega) & =U(-\vec{q},-\Omega) V(\vec{q}, \Omega),
\end{aligned}
$$

necessary to preserve the commutation relations from the input to the output face of the crystal.

Thanks to Eq. (27) we can easily evaluate the PWP result for the biphoton amplitude valid for any gain regime:

$$
\begin{aligned}
\psi\left(\vec{x}, t, \vec{x}^{\prime}, t^{\prime}\right)= & \int \frac{d^{2} \vec{q} d \Omega}{(2 \pi)^{3 / 2}} \int \frac{d^{2} \vec{q}^{\prime} d \Omega^{\prime}}{(2 \pi)^{3 / 2}} \delta\left(\vec{q}+\vec{q}^{\prime}\right) \delta\left(\Omega+\Omega^{\prime}\right) \\
& \times U(\vec{q}, \Omega) V\left(\vec{q}^{\prime}, \Omega^{\prime}\right) e^{i(\vec{q} \cdot \vec{x}-\Omega t)} e^{i\left(\vec{q}^{\prime} \cdot \vec{x}^{\prime}-\Omega^{\prime} t^{\prime}\right)} \\
= & \int \frac{d^{2} \vec{q} d \Omega}{(2 \pi)^{3}} e^{i \vec{q} \cdot\left(\vec{x}-\vec{x}^{\prime}\right)-i \Omega\left(t-t^{\prime}\right)} F(\vec{q}, \Omega)
\end{aligned}
$$

where we introduce the gain function

$$
\begin{aligned}
F(\vec{q}, \Omega) \equiv & U(\vec{q}, \Omega) V(-\vec{q},-\Omega) \\
= & \frac{g e^{i k_{p} l_{c}}}{\Gamma(\vec{q}, \Omega)} \sinh [\Gamma(\vec{q}, \Omega)]\{\cosh [\Gamma(\vec{q}, \Omega)] \\
& \left.+i \frac{\Delta_{\mathrm{pw}}(\vec{q}, \Omega) l_{c}}{2 \Gamma(\vec{q}, \Omega)} \sinh [\Gamma(\vec{q}, \Omega)]\right\},
\end{aligned}
$$

which is strongly peaked around the phase-matching "curve," defined by the equation $\Delta_{\mathrm{pw}}(\vec{q}, \Omega)=0$.

Similarly, the coherence function reads:

$$
G^{(1)}\left(\vec{x}, t, \vec{x}^{\prime}, t^{\prime}\right)=\int \frac{d^{2} \vec{q} d \Omega}{(2 \pi)^{3}} e^{-i \vec{q} \cdot\left(\vec{x}-\vec{x}^{\prime}\right)+i \Omega\left(t-t^{\prime}\right)}|V(\vec{q}, \Omega)|^{2} .
$$

We note that both the biphoton amplitude and the coherence function depend only on the relative coordinates $\vec{\xi}=\vec{x}-\vec{x}^{\prime}$ and $\tau=t-t^{\prime}$, as it is natural to expect for a homogeneous and stationary pump.

By making the limit of $F(\vec{q}, \Omega)$ for $g \rightarrow 0$, of course, we shall obtain the same results as in Sec. II A. We have $\Gamma(\vec{q}, \Omega) \rightarrow i \Delta_{\mathrm{pw}}(\vec{q}, \Omega) l_{c} / 2$, $\cosh [\Gamma(\vec{q}, \Omega)] \rightarrow \cos \left[\Delta_{\mathrm{pw}}(\vec{q}, \Omega) l_{c} / 2\right]$, and $\sinh [\Gamma(\vec{q}, \Omega)] \rightarrow$ $i \sin \left[\Delta_{\mathrm{pw}}(\vec{q}, \Omega) l_{c} / 2\right]$, so that

$$
\begin{aligned}
\lim _{g \rightarrow 0} F(\vec{q}, \Omega) & =g e^{i k_{p} l_{c}} e^{i \frac{\Delta \mathrm{pw}(\vec{q}, \Omega)}{2} l_{c}} \operatorname{sinc}\left(\frac{\Delta_{\mathrm{pw}}(\vec{q}, \Omega) l_{c}}{2}\right) \\
& =e^{i k_{p} l_{c}} \mathcal{V}(\vec{q}, \Omega),
\end{aligned}
$$

and Eq. (15a) for the biphoton amplitude can be retrieved. Similarly,

$\lim _{g \rightarrow 0}|V(\vec{q}, \Omega)|^{2}=g^{2} \operatorname{sinc}\left(\frac{\Delta_{\mathrm{pw}}(\vec{q}, \Omega) l_{c}}{2}\right)=|\mathcal{V}(\vec{q}, \Omega)|^{2}$,

and Eq. (20) is retrieved. This result is in agreement with that found into the previous section. The factor $e^{i k_{p} l_{c}}$ in the right-hand side of Eq. (33b) accounts for the linear phase earned by the pump in crossing the crystal and is the PWP limit of the pump distribution $\overline{\mathcal{A}}_{p}$ in Eq. (14).

Coming back to the expression for the biphoton amplitude for any gain regime, we note that the gain function $F(\vec{q}, \Omega)$ depends on variables $(\vec{q}, \Omega)$ only through the function $\Delta_{\text {pw }}(\vec{q}, \Omega)$ [see Eq. (31)]. For the case of e-oo type I parametric down-conversion considered in this work, it has radial symmetry with respect to $\vec{q}: \Delta_{\mathrm{pw}}(\vec{q}, \Omega)=\sqrt{k_{s}^{2}(\Omega)-q^{2}}+$ $\sqrt{k_{s}^{2}(-\Omega)-q^{2}}-k_{p}$, where $q=|\vec{q}|$. We can therefore evaluate Eq. (30) by means of a Fourier-Hankel transform:

$$
\psi(\xi, \tau)=\int_{-\infty}^{\infty} \frac{d \Omega}{2 \pi} e^{-i \Omega \tau} \int_{0}^{\infty} \frac{d q}{2 \pi} q F(q, \Omega) J_{0}(q \xi),
$$

where $\quad \xi=\left|\vec{x}-\vec{x}^{\prime}\right|, \quad \tau=t-t^{\prime}, \quad$ and $\quad J_{0}(x)=$ $(2 \pi)^{-1} \int_{0}^{2 \pi} e^{i x \sin \theta} d \theta$ is the zero-order Bessel function of the first kind.

We can thus numerically solve Eq. (35) to find the spatiotemporal structure of the biphoton amplitude: the results are reported in the next section.

\section{RESULTS}

In order to obtain quantitative results for the biphoton amplitude's structure, we numerically evaluate Eq. (35) for a type I BBO crystal pumped at $\lambda_{p}=352 \mathrm{~nm}$. We consider the full Sellmeier relations for the refractive indexes [33] to calculate the phase mismatch function $\Delta_{\mathrm{pw}}(\vec{q}, \Omega)$ [Eq. (15c)] without approximations. In order to select an appropriate range of wavelengths to mimic, for example, the finite bandwidth of detection, we introduce a symmetrical frequency filter centered at degeneracy with a super-Gaussian profile.

\section{A. Collinear phase matching, 4-mm crystal}

Our first example is a 4-mm crystal cut at $\theta_{p}=33.436^{\circ}$ for collinear phase matching at degeneracy $\left(\Delta_{0}=2 k_{s}-k_{p}=0\right)$. The results are reported in Fig. 1 for two different values of the gain, $g=10^{-3}$ (upper panels) and $g=3$ (lower panels).

In Fig. 1(a) we plot the section of $|F|$ in the $\left(q_{x}, \Omega\right)$ plane, which is strongly peaked around the phase-matching curve defined by the equation $\Delta_{\mathrm{pw}}(\vec{q}, \Omega)=0$. We remind that $F$ is the function we need to Fourier-Hankel transform in order to obtain the biphoton amplitude, as stated in Eq. (35). The dashed vertical lines represent the full width at half maximum (FWHM) of the frequency filter considered, which in this case is equal to $1.37 \times 10^{15} \mathrm{~Hz}$, corresponding to a wavelength band from 550 to $950 \mathrm{~nm}$.

The two-dimensional cut of the biphoton amplitude for $\xi_{y}=0$ is shown in Fig. 1(b), clearly displaying its X-shaped geometry not separable in space and time. The full threedimensional plot have a radial symmetry in the space domain and has therefore a biconical geometry as shown in Fig. 2. The 


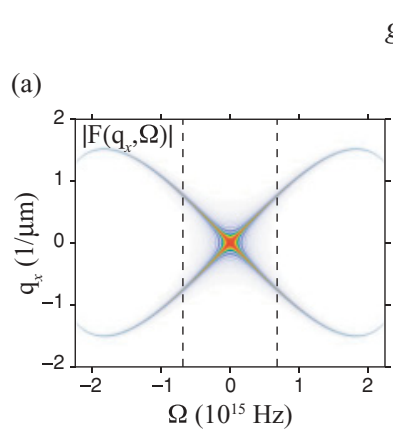

$$
g=10^{-3}
$$

(b) $\tau(\mathrm{fs})$

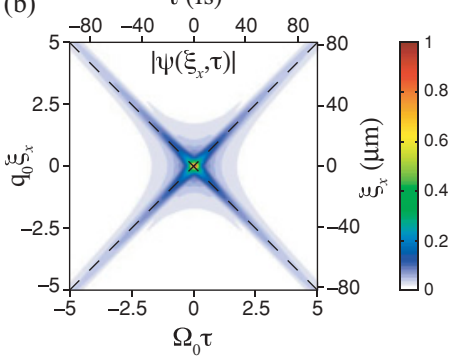

$g=3$
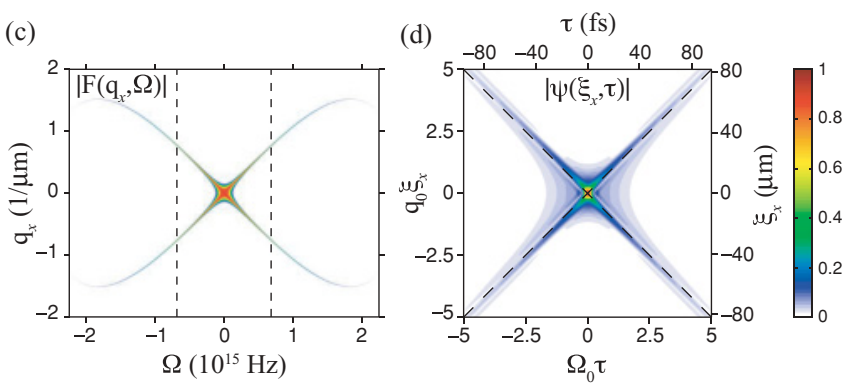

FIG. 1. (Color online) (Left) Section of $|F|$ in the $\left(q_{x}, \Omega\right)$ plane in the low (a) and high (c) gain regime, normalized to its peak value. The dotted lines represent the bandwidth selected by the frequency filter, $\Delta \Omega=1.37 \times 10^{15} \mathrm{~Hz}$. (Right) Two-dimensional plot of the biphoton amplitude (in modulus and normalized to its peak value) in the $\left(\xi_{x}, \tau\right)$ plane for $g=0.001$ (b) and $g=3$ (d), clearly displaying its $\mathrm{X}$-shaped geometry. Note that $\tau$ and $\vec{\xi}$ represent the temporal and spatial relative position of twin photons, respectively. Case of a 4-mm-long type I BBO crystal, pumped at $\lambda_{p}=352 \mathrm{~nm}$, cut at $33.436^{\circ}$ for collinear phase matching at degeneracy.

principal coordinates axes (bottom and left) in Fig. 1(b) report the normalized variables $\Omega_{0} \tau$ and $q_{0} \xi_{x}$, respectively. With respect to these variables the equation for the asymptotes of the

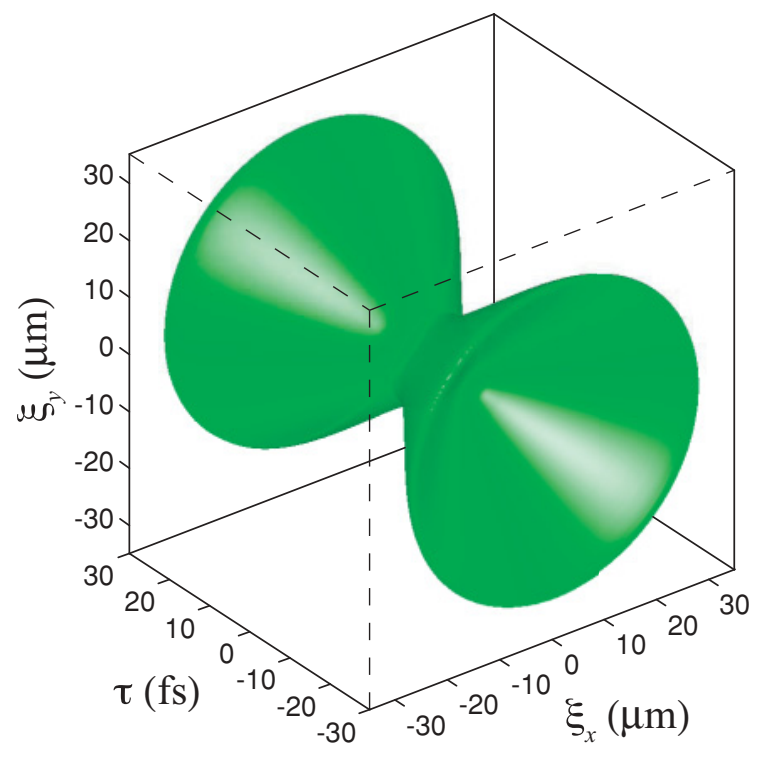

FIG. 2. (Color online) Isosurface representing the full threedimensional structure of the biphoton amplitude (in modulus) for the particular value $\left|\psi\left(\xi_{x}, \xi_{y}, \tau\right)\right|=0.08|\psi(0,0,0)| ; g=0.001$. biphoton amplitude found with the toy model, $q_{0} \xi_{x}= \pm \Omega_{0} \tau$ [see Eq. (17)], simply describes the diagonals represented by the dashed lines in Fig. 1(b), which perfectly fit the legs of the $\mathrm{X}$ structure. The top and right coordinates axes report instead the temporal and spatial relative position of twin photons in physical variables (femtoseconds and micrometers, respectively).

This peculiar $\mathrm{X}$ shape of the biphoton cross correlation is of course reminiscent of the $\mathrm{X}$ wave observed in several nonlinear optical media in the macroscopic regime [34]. In the microscopic case considered here the $\mathrm{X}$ shape of the biphoton amplitude has a completely different interpretation, since it relates the relative spatial localization of twin photons with their temporal separation. Figure 1(b) tells us that if we collect the twin photons from the same near field position, i.e., we consider $\vec{\xi}=\vec{x}-\vec{x}^{\prime}=0$ (e.g., by locating a small pinhole in the near-field of PDC) we are most likely to find them together in time within an uncertainty of few femtoseconds. Conversely, if we collect the twin photons from two separated near field positions $\vec{x}$ and $\vec{x}^{\prime}=\vec{x}+\vec{\xi}$, the temporal correlation peak will split into two separate peaks at $\tau= \pm|\vec{\xi}| q_{0} / \Omega_{0}$, and we are likely to find the two photons delayed by $\pm|\tau|$ within an uncertainty of few femtoseconds.

This particular relationship between the spatial and temporal relative separations of twin photons (as well as the results for the noncollinear case reported in the following sections) can be explained by means of a somehow intuitive picture, which will be illustrated in detail elsewhere [35]. Describing the twin photons as two wave packets centered around the conjugate frequencies $\pm \Omega$, one finds that their temporal separation at the crystal exit face is determined by their different group velocities as imposed by the group velocity dispersion, whereas their spatial separation is determined by diffraction. The phase-matching mechanism (the conservation of longitudinal momentum) imposes a precise relationship between group velocity dispersion and diffraction that can be turned into a relationship between the temporal and spatial separation of twin photons [35].

The results for the high gain are reported in Figs. 1(c) and 1(d). We note that there are no significant differences between the two gain regimes, as the function $F$, and therefore the biphoton amplitude, has basically the same structure, with the only exception being the small oscillations that can be seen in Fig. 1(a). These oscillations can be explained recalling that in the low gain limit the hyperbolic functions that define $F$ can be approximated by the corresponding trigonometric functions, with the characteristic oscillating behavior.

We remark that in type II parametric down-conversion, instead, there is a significant difference between the low and the high gain regime. In that case, in the low gain regime the correlation assumes a V-shaped structure, asymmetric with respect to time (like a halved $\mathrm{X}$ ), with the asymmetry originating from the signal-idler group velocity mismatch. For high gain, instead, the symmetric X geometry of type I PDC is recovered [23].

A remarkable characteristic of the nonseparable structure of the biphoton amplitude is the unusual small width of the correlation peak, which corresponds to an extreme relative localization of twin photons in space and time. In Fig. 3 we plot cuts of the the coincidence rate $|\psi|^{2}$ along the temporal axis 


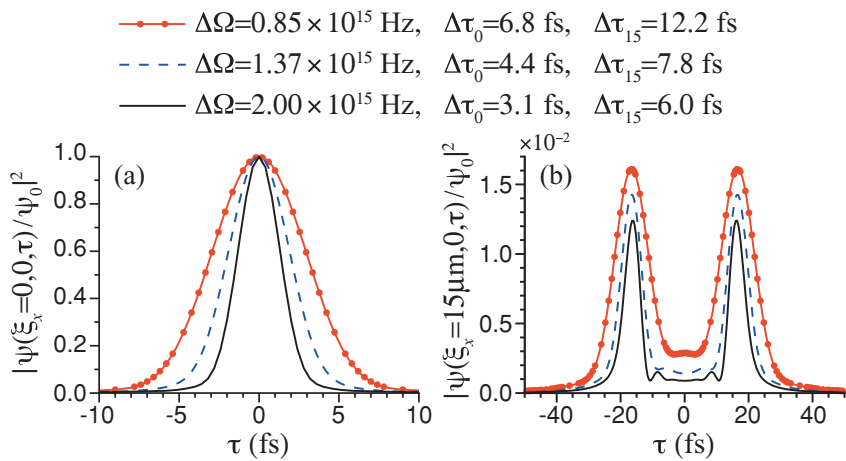

FIG. 3. (Color online) (a) Cut of the coincidence rate along the temporal axis, $|\psi(0,0, \tau)|^{2}$, showing the temporal correlation of twin photons collected from the same near-field position $(\vec{\xi}=0)$ for different frequency filter bandwidths. (b) Same plot as in (a) for $|\vec{\xi}|=15 \mu \mathrm{m}$. The values in the legend correspond to the FWHM of the frequency filter $(\Delta \Omega)$, the FWHM of the temporal coincidence rate for $\vec{\xi}=0\left(\Delta \tau_{0}\right)$, and the FWHM of one peak of the temporal coincidence rate for $|\vec{\xi}|=15 \mu \mathrm{m}\left(\Delta \tau_{15}\right)$. The coincidence rates are normalized to $\psi_{0} \equiv \psi(0,0,0) ; g=0.001$, other parameters as in Fig. 1.

for $\vec{\xi}=0$ (a), and $|\vec{\xi}|=15 \mu \mathrm{m}$ (b), and for different widths of the frequency filter. When photons are collected at the same near-field position we have a single temporal peak [Fig. 3(a)], while when $\vec{\xi} \neq 0$ we observe two symmetric temporal peaks [Fig. 3(b)]. In both cases the uncertainty on the arrival time of the second photon with respect to the first one is on the order of few femtoseconds and is inversely proportional to the bandwidth of the temporal filter used.

As exhaustively explained in Ref. [22], to achieve this extreme temporal localization of twin photons, we need to resolve their near-field relative position. In this case indeed the correlation time is determined by the inverse of the full ultrabroad bandwidth of the PDC phase matching or in practice by the bandwidth of the frequency filter. On the contrary, if we do not resolve the near-field relative position of twin photons, the correlation time is determined by the inverse of the bandwidth of $F(\vec{q}, \Omega)$ at fixed $\vec{q}$, which depends on the crystal length and for our example of a 4-mm crystal is on the order of $10^{13}-10^{14} \mathrm{~Hz}$, therefore one or two orders of magnitude smaller (see next section for a more detailed discussion).

In Fig. 4 we plot the spatial profile of the coincidence rate, when the relative arrival time of twin photons is resolved at $\tau=0$. In this case we observe a correlation length on the order of few micrometers that also scales inversely with the bandwidth of the temporal frequency filter considered. This is the analog of the effect shown in Ref. [22], where we found that the correlation time of twin photons scales inversely with the width of a spatial filter put in the far field. The correspondence between the temporal bandwidth and the spatial correlation length, or, vice versa, the one between the spatial bandwidth and the correlation time, is due to the characteristic, not separable, relationship between spatial frequencies and temporal frequencies imposed by the phase-matching mechanism.

To go deeper into the understanding of $\mathrm{X}$ entanglement we shall examine different configuration for the PDC process,

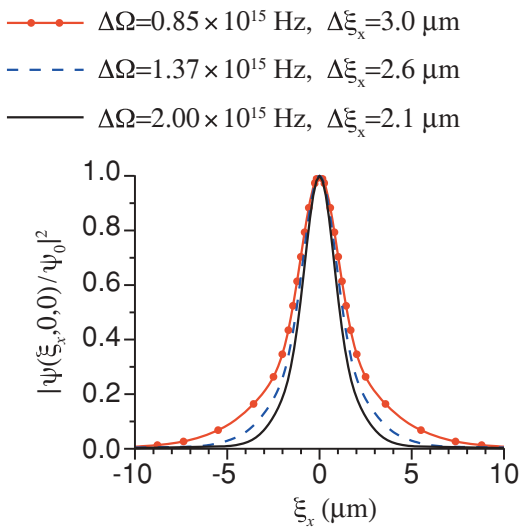

FIG. 4. (Color online) (a) Cut of the coincidence rate along the spatial axis, $\left|\psi\left(\xi_{x}, 0,0\right)\right|^{2}$, for different temporal frequency filter widths, showing the spatial correlation of twin photons. $\Delta \Omega$ and $\Delta \xi_{x}$ represent the FHWM of the frequency filter and of the coincidence rate, respectively. Also in this case the width of the correlation peak scales inversely to the width of the frequency filter (see text for details).

such as different crystal lengths and tuning angles. We shall show the intrinsic relationship between the phase-matching mechanism and the biphoton amplitude's structure and that the nonfactorizability in space and time of the biphoton amplitude is the key element to completely understand and thus manipulate the biphoton properties.

\section{B. Biphoton cross correlation for different crystal lengths}

In the previous section we explained how in order to achieve an extreme temporal localization of twin photons in the femtosecond range, it is necessary to resolve their near-field relative positions. Nevertheless, this kind of correlation can in principle be reached also with usual detection techniques, i.e., by collecting photons from the whole cross section, without discriminating their positions. The quantity measured is then the integrated coincidence rate

$$
C(\tau) \propto \int d^{2} \vec{\xi}|\psi(\vec{\xi}, \tau)|^{2} .
$$

However, in this case, a femtosecond localization is reached only when a very thin crystal (tens of microns) is employed, which implies a very low down-conversion efficiency. On the other hand, we shall show that if we resolve the twin photons position in the near field, we can reach a temporal localization on the order of few femtoseconds even for a long crystal. The crystal length influences the PDC process because for a thin crystal the phase-matching condition $\Delta_{\mathrm{pw}}(\vec{q}, \Omega)=0$ is less restrictive: also the modes that gives rather large values of $\Delta_{\mathrm{pw}}$ are amplified. Therefore, for a thin crystal, the function $F(\vec{q}, \Omega)$ is less peaked around the perfect phase-matching curve, and we expect that while the cross-correlation peak maintains its width, the tails of the $\mathrm{X}$ structure become more visible as the crystal becomes thicker.

Figure 5 shows the effects of the crystal length on the structure of the biphoton amplitude. All other parameters are not varied (in particular $g=10^{-3}$ ). The upper panels plot 
(a) $l_{c}=0.1 \mathrm{~mm}$
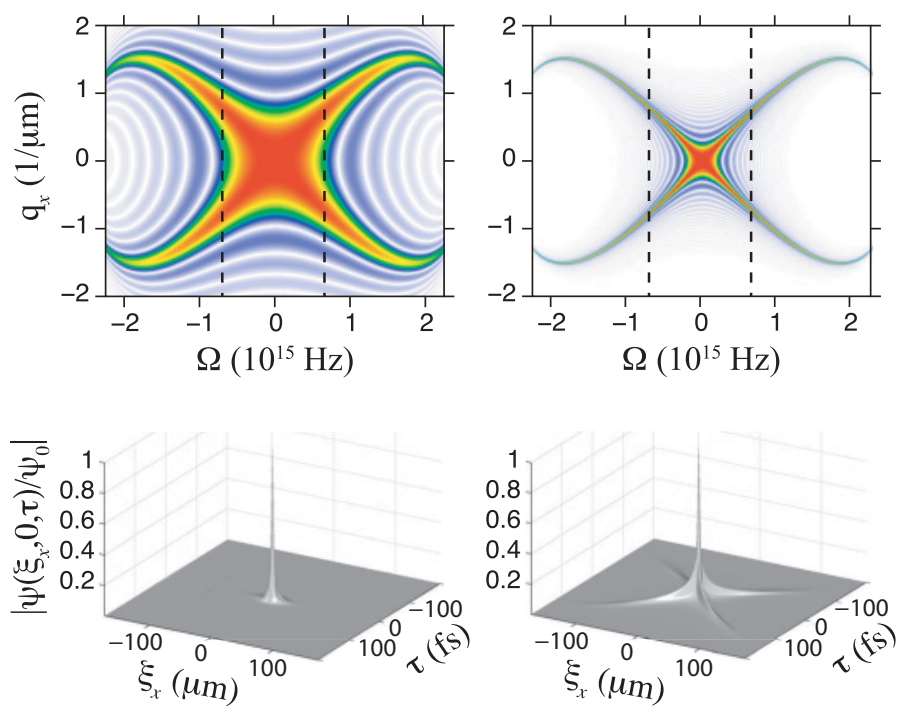

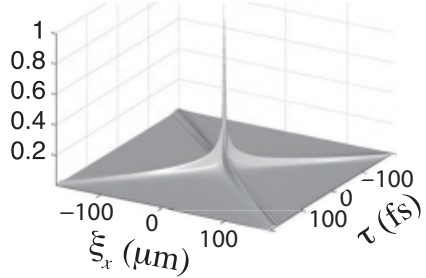

(c) $\quad l_{c}=4 \mathrm{~mm}$

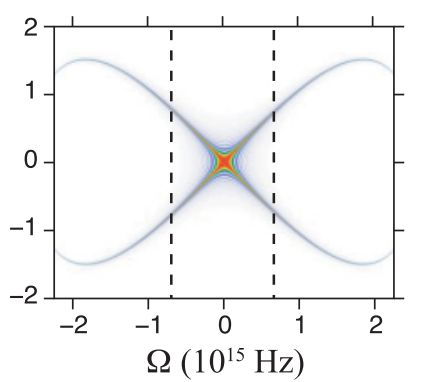

(d) $l_{c}=12 \mathrm{~mm}$
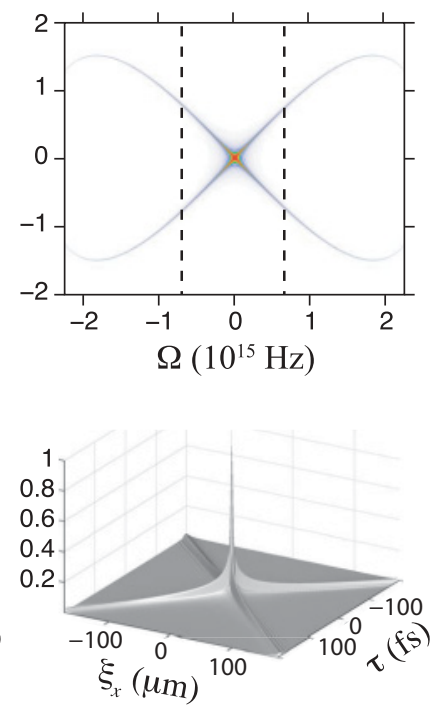
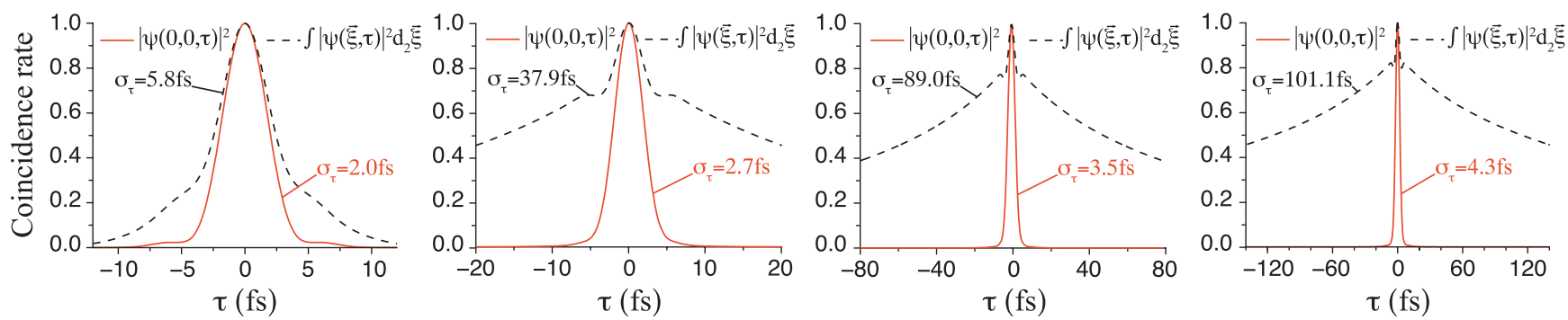

FIG. 5. (Color online) Effect of the crystal length on the biphoton amplitude. The first row reports $\left|F\left(q_{x}, \Omega\right)\right|$ for different crystal lengths: (a) $0.1 \mathrm{~mm}$, (b) $1 \mathrm{~mm}$, (c) $4 \mathrm{~mm}$, and (d) $12 \mathrm{~mm}$. The dotted vertical lines represents the FWHM of the frequency filter considered. As expected, $F$ develops a structure that becomes thinner increasing the crystal length. The central row shows the corresponding biphoton amplitude $\left|\psi\left(\xi_{x}, \tau\right)\right|$. While the width of the peak remains unchanged, it is evident the increasing of the tails visibility as the crystal becomes longer. The last row compares the coincidence rate when twin photons are collected from the same near-field position (red solid line), with the integrated coincidence rate obtained without discriminating their positions (black dashed line). To evaluate the difference we calculate for each case the standard deviation $\sigma_{\tau}$ : while for $|\psi(0,0, \tau)|^{2}$ the temporal correlation is almost the same for each crystal length, for the integrated coincidence rate it increases up to 20 times. $\theta_{p}=33.436^{\circ}, \lambda_{p}=352 \mathrm{~nm}, g=10^{-3}$.

$\left|F\left(q_{x}, \Omega\right)\right|$, with the filter width being represented by the dotted vertical lines, while the central panels show the corresponding biphoton amplitudes $\left|\psi\left(\xi_{x}, \tau\right)\right|$.

For a very thin crystal, Fig. $5(\mathrm{a}), l_{c}=0.1 \mathrm{~mm}$, the typical $\infty$-shaped structure of the gain function $F$, in the $\left(q_{x}, \Omega\right)$ plane, can be hardly appreciated because the phase-matching bandwidth becomes extremely broad. If we restrict to the region delimitated by the frequency filter (between the dashed lines), no structure is actually visible. As a consequence the biphoton amplitude in the $\left(\xi_{x}, \tau\right)$ plane shows basically a single peak and the $\mathrm{X}$ structure of the correlation is absent.

However, by increasing the crystal length, the phasematching mechanism starts to impose a well-defined relation between $\vec{q}$ and $\Omega$ of the down-converted modes, which shows up in the well-defined and narrow shape of the gain function $F$ in the $\left(q_{x}, \Omega\right)$ plane. In turns, the biphoton correlation starts to develop a defined sharp structure, with the tails of the $\mathrm{X}$ structure becoming more and more visible as the crystal length increases.
It is important to stress that the width of the central peak of the $\mathrm{X}$ structure remains the same for all the crystal lengths, both in the temporal and in the spatial domains. Actually the intercepted bandwidth is the same in all cases, as it corresponds to the portion of the PDC emission selected by the frequency filter. This means that the $\mathrm{X}$-shaped structure of the biphoton amplitude allows to obtain ultrabroadband biphotons also for long crystal, which may in principle result in an higher down-conversion efficiency. However, even if the total number of down-converted photons increases with the crystal length, the number of twin photons localized within few femtoseconds and few microns (namely the ones populating the central peak of $\psi$ ) does not necessarily increase proportionally. In fact, increasing the crystal length more and more twin photons will appear separated in space and time at the output face of the nonlinear medium, populating the tails of the $\mathrm{X}$ structure. This is clearly shown by Fig. 5 (central row), where the tails of the $\mathrm{X}$ structure become more and more visible as the crystal length increases. Further quantitative work on this subject, which is beyond the scope of this work, is necessary in 
order to obtain the precise way in which these two factors balance. Nevertheless, we notice that using a rather long crystal in combination with a spatially resolved measurement offers a larger flexibility with respect to the use of a short crystal. In the first case, indeed, operating on the spatial degrees of freedom (namely by changing the dimensions and positions of near-field detectors) opens the possibility of changing the temporal localization of biphotons by orders of magnitude.

In the lower panels of Fig. 5 we compare the coincidence rate when taking photons from the same near-field position $\left(|\psi(0,0, \tau)|^{2}\right.$, red solid line $)$, with the integrated coincidence rate [Eq. (36), black dashed line] obtained when collecting all the photons over the beam cross section, without discriminating their position. In particular, we calculate the standard deviation $\sigma_{\tau}$ of the two distributions, where for a generic distribution $h(\tau)$, with zero mean, we define:

$$
\sigma_{\tau} \equiv \sqrt{\frac{\int \tau^{2} h(\tau) d \tau}{\int h(\tau) d \tau}} .
$$

As expected, for a very thin crystal [Fig. 5(a), lower panel] the two distributions are very similar, because the width of $F(\vec{q}, \Omega)$ at fixed $\vec{q}$ is as broad as the frequency filter. Increasing the crystal length, $F(\vec{q}, \Omega)$ becomes thinner and thinner, and the standard deviation of the integrated coincidence rate increases up to $101.1 \mathrm{fs}$, while $\sigma_{\tau}$ of the coincidence rate for $\vec{\xi}=0$ is still a few femtoseconds. Therefore the X-shaped structure of the biphoton amplitude is the key element to access an ultrabroad bandwidth source of entangled photons also for long crystals.

\section{Biphoton cross correlation at different crystal tuning angles}

In the previous sections we have seen that in order to obtain a narrow temporal localization of twin photons, it is necessary to resolve their near-field position, as a consequence of the nonseparable character of the space-time correlation. Conversely, in order to obtain a narrow spatial localization, one should resolve temporally the photons arrival time within a few femtoseconds, which is indeed highly unpractical due to the typical slow response time of detectors.

In this section we shall explore the close relationship between the phase-matching properties and the biphoton amplitude's structure. We shall show situations where the state becomes factorizable with respect to space and time, and an extremely narrow spatial and temporal localization can be achieved independently, i.e., a femtosecond temporal localization can be achieved without the need of controlling the spatial degrees of freedom and vice versa.

In particular, we change the tuning angle $\theta_{p}$ between the pump propagation direction and the principal axis of the crystal, from the collinear case [Fig. 6(a)], to strongly non-collinear phase matching. In such conditions, the PDC emission occurs over a narrow ring of transverse wave vectors of radius $\bar{q}$, over a very large temporal bandwidth [Fig. 6(d)].

In Fig. 6 we report the structure of the biphoton amplitude for the different tuning angles considered. The upper panels plot the function $\left|F\left(q_{x}, \Omega\right)\right|$, while the lower panels show the corresponding biphoton correlation in the $\left(\xi_{x}, \tau\right)$ plane. As the tuning angle increases, there is a transition from the $\mathrm{X}$ structure to a cigarlike structure, which is separable in space and time. In these conditions a narrow localization of biphotons
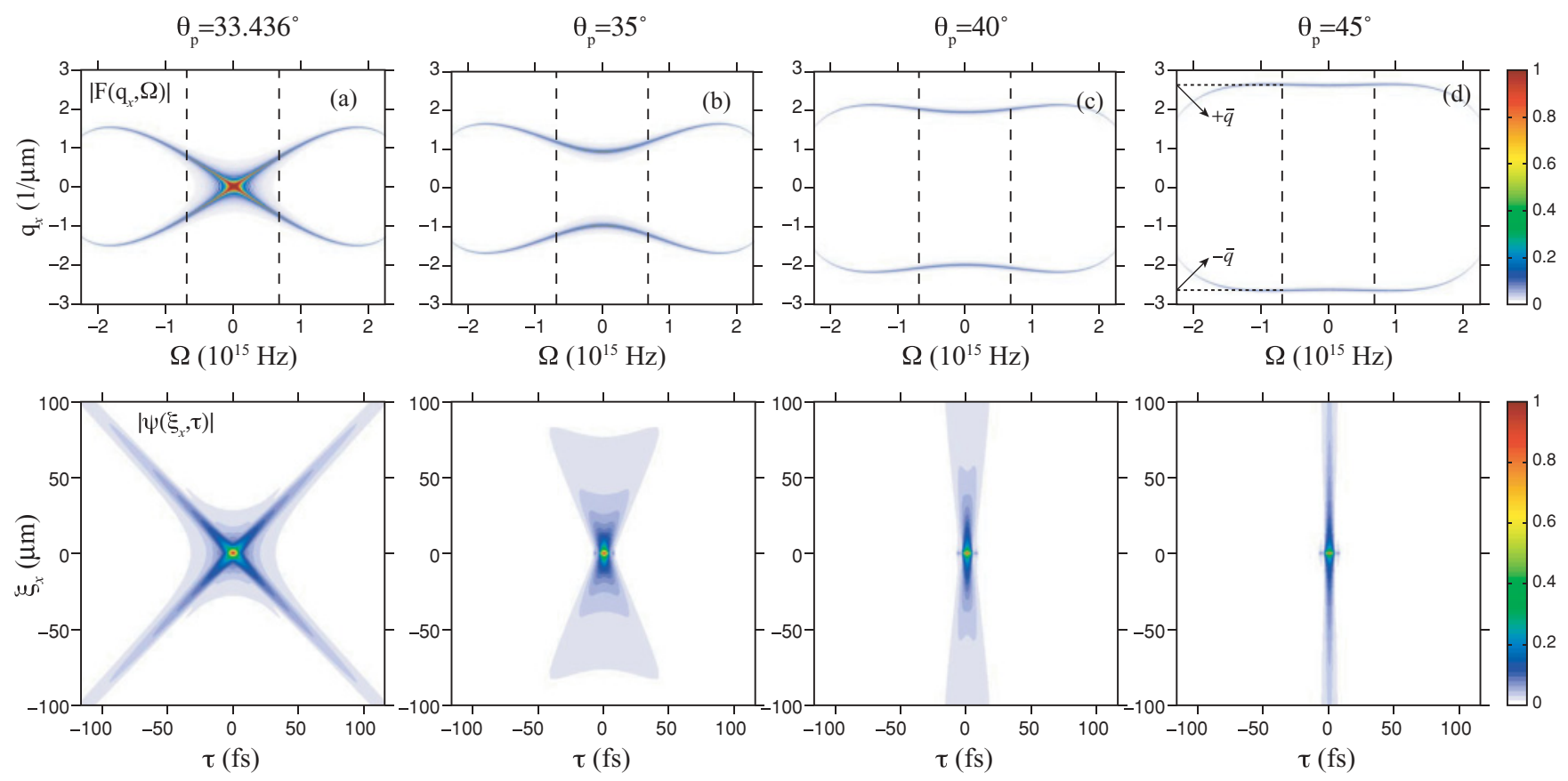

FIG. 6. (Color online) Transition from a nonseparable to a separable state. (Upper panels) Plot of $\left|F\left(q_{x}, \Omega\right)\right|$ for different tuning angles from the collinear to the strongly noncollinear case: (a) $\theta_{p}=33.436^{\circ}$, (b) $35^{\circ}$, (c) $40^{\circ}$, and (d) $45^{\circ}$. In the lower panels we report the corresponding $2 \mathrm{D}$ cut of the biphoton amplitude $\left|\psi\left(\xi_{x}, \tau\right)\right|$. Increasing the tuning angle we see a transition from the nonseparable $\mathrm{X}$ geometry to a separable cigarlike geometry: in this last case an extreme localization of twin photons can be achieved both in space and time independently. We consider $l_{c}=4 \mathrm{~mm}, g=10^{-3}, \lambda_{p}=352 \mathrm{~nm}$. 

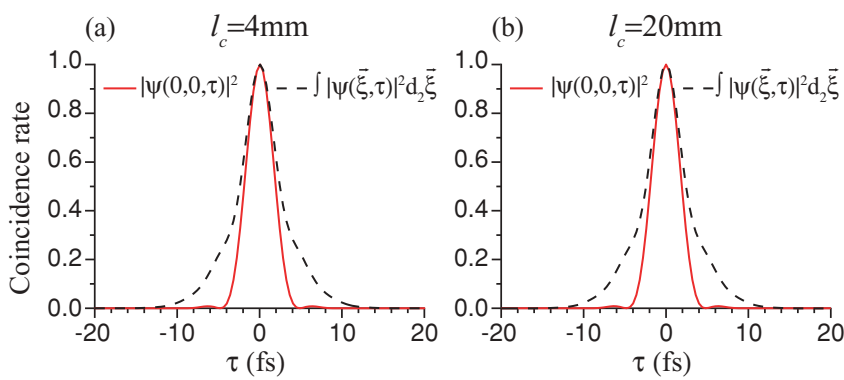

FIG. 7. (Color online) Case of strongly noncollinear phase matching $\theta_{p}=45^{\circ}$. (a) Temporal profile of the coincidence rate when photons are detected from the same position (red solid line) and when their positions are not resolved (black dashed line) for $l_{c}=4 \mathrm{~mm}$. The width of the correlation peak is basically the same. (b) Same as in (a) but for $l_{c}=20 \mathrm{~mm}$, showing no appreciable changes in the plot. Even if the biphoton amplitude is separable in space and time, the temporal correlation of twin photons is independent from the crystal length.

can be achieved both in space and time independently: even without discriminating the photons position, it is in principle possible to achieve a few fs temporal localization.

Actually the integrated coincidence rate $C(\tau)$, obtained collecting the photons from the whole beam cross section, without discriminating their positions, has almost the same width of the coincidence rate obtained when collecting twin photons from the same near-field position, as shown in Fig. 7(a). On the contrary, if we consider the same comparison for the collinear phase matching, the difference between the correlation times is more than one order of magnitude, as shown in the lower panel of Fig. 5(c).

We note that in this case of strongly noncollinear phase matching, even if the biphoton amplitude becomes separable in space and time, the temporal correlation of twin photons does not broaden with the crystal length, as shown by comparing Figs. 7(a) and 7(b). This may appear surprising, because in conditions of collinear or quasicollinear phase matching the temporal correlation measured by a bucket detector broadens with the crystal length, as shown by Fig. 5 (lower row). This depends on the narrowing of the gain function $F$ in the $(q, \Omega)$ domain with increasing crystal length, which in turns implies a narrower frequency bandwidth at fixed $q$. Since this bandwidth determines the temporal localization of twin photons measured by mean of a bucket detector [22], in this case the temporal correlation broadens.

In the present case of strongly noncollinear phase matching instead, this broadening of the temporal correlation does not occur. The reason is that the phase-matching curve in the $(q, \Omega)$ plane is almost a straight line parallel to the $\Omega$ axis, which means that the narrowing of the gain function $F$ occurs only in the $q$ direction and does not affect the frequency bandwidth.

In Fig. 8 we show the same comparison reported in Fig. 7 for the spatial domain: in this case the coincidence rate for $\tau=0$ and its temporal integral have exactly the same profile (some differences however become appreciable when one plots the figure on a larger scale), characterized by an ultranarrow peak with a FWHM of only $0.9 \mu \mathrm{m}$. This is a rather striking result with respect to the typical spatial localization of twin photons predicted so far [25]. This is on the order of tens of microns

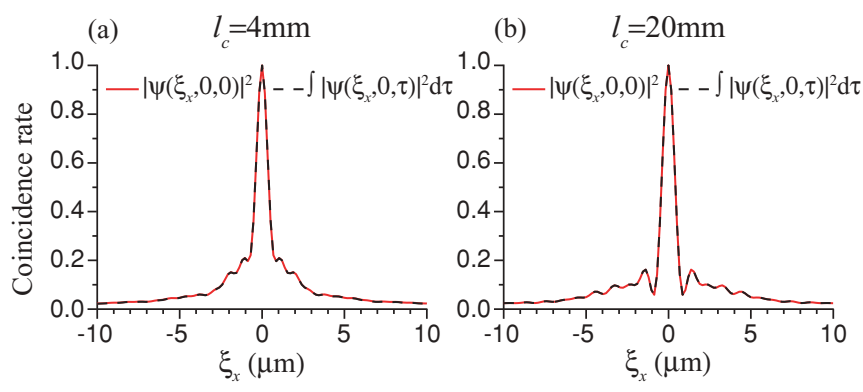

FIG. 8. (Color online) Case of strongly noncollinear phase matching $\theta_{p}=45^{\circ}$. Spatial profile of the coincidence rate. Superimposed are the two curves obtained by resolving the photon relative arrival time ( $\tau=0$, red solid line) and by not resolving it, i.e., by integrating over long time intervals (black dashed line) for $l_{c}=4 \mathrm{~mm}$ (a) and $l_{c}=20 \mathrm{~mm}(\mathrm{~b})$.

in collinear configurations, both in the near field (integrating over long time) and in the far field [36,37].

In the noncollinear case analyzed here, a narrow spatial correlation peak arises because of the particular spatial geometry of the emission (a large ring of transverse wave vectors). In analogy to the case of the pulsed Bessel beams [38], characterized by an intensity spectrum in the $(\vec{q}, \Omega)$ domain with the same geometry of $F(\vec{q}, \Omega)$ reported in Fig. $6(\mathrm{~d})$, we expect that the biphoton amplitude as a function of the spatial relative near-field coordinates, is well described by the Bessel function $J_{0}(\bar{q} \xi)$. Due to the large value of $\bar{q}=2.62 \mu \mathrm{m}^{-1}$, the spatial correlation is characterized by an ultranarrow peak.

Also in the spatial domain the twin photons localization is independent from the crystal length. Actually, even if there is some difference between the spatial profiles of the coincidence rate in Figs. 8(a) and 8(b), the width of the central peak is exactly the same. Also in this case the reason is to be searched in the geometry of phase matching. The extreme localization of the spatial correlation is determined by the large value of $\bar{q}$. Since by increasing the crystal length the gain function becomes narrower, but the value of $\bar{q}$ does not change, also the spatial localization remains the same.

We can go deeper in the analogy with the pulsed Bessel beams by considering the paraxial and quadratic dispersion approximation, thanks to which the phase mismatch function reduces to [24]

$$
\Delta_{\mathrm{pw}}(\vec{q}, \Omega) l_{c}=\Delta_{0} l_{c}-\frac{q^{2}}{q_{0}^{2}}+\frac{\Omega^{2}}{\Omega_{0}^{2}},
$$

where $\Delta_{0}=2 k_{s}-k_{p}$ is the collinear phase mismatch. Highly noncollinear phase matching is achieved for large positive values of $\Delta_{0}$. For example for $\theta_{p}=40^{\circ}, \Delta_{0}=260 \mathrm{~mm}^{-1}$ while for $\theta_{p}=45^{\circ}, \Delta_{0}=462 \mathrm{~mm}^{-1}$.

For $\theta_{p}=45^{\circ}$ [Fig. 6(d)], in a broad range of frequencies around degeneracy corresponding to $\Omega \ll \Omega_{0} \sqrt{\Delta_{0} l_{c}}$, perfect phase matching is achieved basically for a single value of $q=\bar{q} \approx q_{0} \sqrt{\Delta_{0} l_{c}}=\sqrt{k_{s} \Delta_{0}}$ (notice that it does not depend on $l_{c}$ ). Moreover, in this case the PDC emission occurs in a very narrow spatial bandwidth $\delta_{q} \approx q_{0} / \sqrt{\Delta_{0} l_{c}}=l_{c}^{-1} \sqrt{k_{s} / \Delta_{0}}$ around $\bar{q}$. In other words the PDC emission takes place over a narrow ring of spatial frequencies for a broad range of temporal 

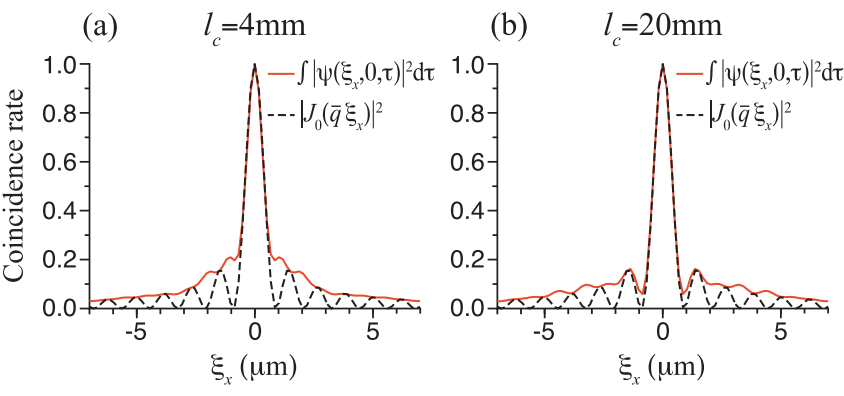

FIG. 9. (Color online) Comparison between the spatial correlation function $\int d \tau\left|\psi\left(\xi_{x}, 0, \tau\right)\right|^{2}=\left|\psi\left(\xi_{x}, 0,0\right)\right|^{2}$ and the square modulus of the Bessel function $J_{0}\left(\bar{q} \xi_{x}\right)$, with $\bar{q}$ such that $\Delta_{\mathrm{pw}}(\bar{q}, 0)=$ 0 , for $l_{c}=4 \mathrm{~mm}$ (a) and $l_{c}=20 \mathrm{~mm}$ (b). All functions are normalized to their peak value.

frequencies. ${ }^{1}$ In these conditions the biphoton amplitude can be approximated as

$$
\begin{aligned}
\psi(\xi, \tau) & =\int_{-\infty}^{\infty} \frac{d \Omega}{2 \pi} e^{-i \Omega \tau} \int_{0}^{\infty} \frac{d q}{2 \pi} q F(q, \Omega) J_{0}(q \xi) \\
& \approx J_{0}(\bar{q} \xi) \int_{-\infty}^{\infty} \frac{d \Omega}{2 \pi} e^{-i \Omega \tau} \int_{0}^{\infty} \frac{d q}{2 \pi} q F(q, \Omega) \\
& =J_{0}(\bar{q} \xi) G(\tau)
\end{aligned}
$$

where

$$
G(\tau)=\int_{-\infty}^{\infty} \frac{d \Omega}{2 \pi} e^{-i \Omega \tau} \int_{0}^{\infty} \frac{d q}{2 \pi} q F(q, \Omega)
$$

and we used the fact that $F(q, \Omega)$ is an extremely narrow function peaked around $\bar{q}$, so that the Bessel function $J_{0}$ can be extracted from the integral at the right-hand side of Eq. (39a).

From the expression in Eq. (39c) we clearly see (i) the factorizable character of the state in space and time and (ii) the Bessel-like shape of the spatial correlation, exactly as expected from the analogy with the pulsed Bessel beams. As for the collinear phase matching, where the X-shaped biphoton correlation can be regarded as the microscopic counterpart of the nonlinear $\mathrm{X}$ waves, in this case of strongly noncollinear phase matching, we can consider the biphoton correlation as the microscopic analog of the pulsed Bessel beams.

Since we know that the quadratic dispersion approximation is a quite crude approximation over the large frequency bandwidth considered in this work, in order to support the analogy developed so far, we directly compare the spatial profile of the coincidence rate and the Bessel function $J_{0}\left(\bar{q} \xi_{x}\right)$. The result is reported in Fig. 9, for two different crystal lengths. The characteristic oscillations of the Bessel function are not exactly reproduced in the spatial correlation, but we note that $\int d \tau\left|\psi\left(\xi_{x}, 0, \tau\right)\right|^{2}$ matches well the envelope of $\left|J_{0}\right|^{2}$, and in particular the width of the peak is exactly the same. Moreover, as the crystal length increases, $\left|J_{0}\right|^{2}$ fits better the coincidence

\footnotetext{
${ }^{1}$ Note that a narrow ring of spatial frequencies $\vec{q}$ does not necessarily imply a narrow angular bandwidth of emission, because for a large temporal bandwidth, emission takes place for each $\Omega$ at an angle $\theta(\Omega)=\arcsin \left[\bar{q} c_{0} /\left(\omega_{p} / 2+\Omega\right)\right]$ that varies from $13.5^{\circ}$ to $23.2^{\circ}$ (external angles) for $\Delta \Omega=1.37 \times 10^{15} \mathrm{~Hz}$.
}

rate, due to the fact that the gain function in the $\left(q_{x}, q_{y}\right)$ plane approaches an ideal ring of infinitesimal thickness.

We note that, as in the previous sections, the structure and the characteristics of the biphoton amplitude are determined by the phase-matching properties. The nature of this relationship can be better understood by means of the intuitive picture reported in Ref. [35].

\section{CONCLUSIONS}

We have shown that a complete description of the biphoton entangled state produced in the parametric down-conversion process must be carried out in a fully spatiotemporal framework.

In conditions of collinear or quasicollinear phase matching the structure of the biphoton correlation turns out to be nonseparable in space and time and governed by a peculiar $\mathrm{X}$ geometry. This particular structure is imposed, at the microscopic level, by the phase-matching mechanism governing the process. For this reason the full spatiotemporal description of the biphoton state, together with the study of the relationship between its structure and the phase-matching mechanism, are the key elements to completely describe and thus manipulate the biphotons properties. We have shown that it is possible to manipulate the biphoton properties by choosing the correct detection technique that takes into account the nonseparability in space and time. In particular we have shown that by discriminating the near-field position of twin photons it is possible to access the ultrabroad phase-matching bandwidth, which implies a correlation time of biphotons on the order of few femtoseconds. This result is almost independent on the length of the nonlinear medium, while with usual detection techniques (that is, by collecting photons from the whole beam section) the correlation time increases with the crystal length.

We have shown that by properly manipulating the phasematching conditions it is possible to modify the structure of the entangled state. In particular by changing the crystal tuning angle so to achieve strongly noncollinear phase matching, we see a transition from a nonseparable $X$ geometry to a fully separable geometry in space and time. This latter situation could be indeed very interesting for applications because an extremely broad temporal frequency bandwidth can be achieved independently from the spatial measurement, i.e., twin photons can be localized in time within few femtoseconds without the need of resolving their positions. The same situation arises in space, where an ultranarrow spatial localization, on the order of $1 \mu \mathrm{m}$, can be achieved without the need of controlling the temporal degrees of freedom, thanks to the Bessel-like form of the spatial correlation. This latter result can be of great importance for those quantum imaging applications, such as correlated imaging or quantum lithography, where the spatial localization of biphotons limits the spatial resolution achievable in the schemes.

\section{ACKNOWLEDGMENTS}

We thank M. Clerici, L. A. Lugiato, O. Jedrkiewicz, and P. Di Trapani for useful discussions. We acknowledge the 
financial support of the Future and Emerging Technologies (FET) programme within the Seventh Framework Programme for Research of the European Commission, under the FETOpen grant agreement HIDEAS, number FP7-ICT-221906.

\section{APPENDIX A}

In this appendix we solve with a perturbative approach the propagation equation for the field, Eq. (4), the gain $g$ being the perturbative parameter, that we assume $\ll 1$. We start formally integrating Eq. (4) from $z=0$ to a generic $z$ :

$$
\begin{aligned}
& \hat{a}(\vec{q}, \Omega, z)-\hat{a}(\vec{q}, \Omega, 0) \\
& =\frac{g}{l_{c}} \int_{0}^{z} d z^{\prime} \int \frac{d \vec{q}^{\prime} d \Omega^{\prime}}{(2 \pi)^{3 / 2}} \bar{\alpha}_{p}\left(\vec{q}+\vec{q}^{\prime}, \Omega+\Omega^{\prime}\right) \\
& \quad \times \hat{a}^{\dagger}\left(\vec{q}^{\prime}, \Omega^{\prime}, z^{\prime}\right) e^{-i \Delta\left(\vec{q}, \Omega, \vec{q}^{\prime}, \Omega^{\prime}\right) z^{\prime}} .
\end{aligned}
$$

We can find a similar equation for $\hat{a}^{\dagger}$, and substituting recursively the expressions for the fields, we obtain:

$$
\begin{aligned}
\hat{a}(\vec{q}, \Omega, z) \\
=\hat{a}(\vec{q}, \Omega, 0)+\frac{g}{l_{c}} \int_{0}^{z} d z^{\prime} \int \frac{d \vec{q}^{\prime} d \Omega^{\prime}}{(2 \pi)^{3 / 2}} \bar{\alpha}_{p}\left(\vec{q}+\vec{q}^{\prime}, \Omega+\Omega^{\prime}\right) \\
\quad \times \hat{a}^{\dagger}\left(\vec{q}^{\prime}, \Omega^{\prime}, 0\right) e^{-i \Delta\left(\vec{q}, \Omega, \vec{q}^{\prime}, \Omega^{\prime}\right) z^{\prime}}+\frac{|g|^{2}}{l_{c}^{2}} \int_{0}^{z} d z^{\prime} \int \frac{d \vec{q}^{\prime} d \Omega^{\prime}}{(2 \pi)^{3 / 2}} \\
\quad \times \bar{\alpha}_{p}\left(\vec{q}+\vec{q}^{\prime}, \Omega+\Omega^{\prime}\right) e^{-i \Delta\left(\vec{q}, \Omega, \vec{q}^{\prime}, \Omega^{\prime}\right) z^{\prime}} \int_{0}^{z^{\prime}} d z^{\prime \prime} \\
\quad \times \int \frac{d \vec{q}^{\prime \prime} d \Omega^{\prime \prime}}{(2 \pi)^{3 / 2}} \bar{\alpha}_{p}^{*}\left(\vec{q}^{\prime}+\vec{q}^{\prime \prime}, \Omega^{\prime}+\Omega^{\prime \prime}\right) \hat{a}\left(\vec{q}^{\prime \prime}, \Omega^{\prime \prime}, 0\right) \\
\quad \times e^{i \Delta\left(\vec{q}^{\prime}, \Omega^{\prime} ; \vec{q}^{\prime \prime}, \Omega^{\prime \prime}\right) z^{\prime \prime}}+\cdots
\end{aligned}
$$

Up to first order in $g$, and for $z=l_{c}$, we therefore get:

$$
\begin{aligned}
\hat{a}\left(\vec{q}, \Omega, l_{c}\right) & \\
= & \hat{a}(\vec{q}, \Omega, 0)+\frac{g}{l_{c}} \int_{0}^{l_{c}} d z \int \frac{d \vec{q}^{\prime} d \Omega^{\prime}}{(2 \pi)^{3 / 2}} \bar{\alpha}_{p}\left(\vec{q}+\vec{q}^{\prime}, \Omega+\Omega^{\prime}\right) \\
& \times \hat{a}^{\dagger}\left(\vec{q}^{\prime}, \Omega^{\prime}, 0\right) e^{-i \Delta\left(\vec{q}, \Omega, \vec{q}^{\prime}, \Omega^{\prime}\right) z} .
\end{aligned}
$$

By introducing the change of variables $z \rightarrow s=z / l_{c}$, we obtain:

$$
\begin{aligned}
\hat{a}\left(\vec{q}, \Omega, l_{c}\right) \\
=\hat{a}(\vec{q}, \Omega, 0)+g \int_{0}^{1} d s \int \frac{d \vec{q}^{\prime} d \Omega^{\prime}}{(2 \pi)^{3 / 2}} \bar{\alpha}_{p}\left(\vec{q}+\vec{q}^{\prime}, \Omega+\Omega^{\prime}\right) \\
\quad \times \hat{a}^{\dagger}\left(\vec{q}^{\prime}, \Omega^{\prime}, 0\right) e^{-i \Delta\left(\vec{q}, \Omega, \vec{q}^{\prime}, \Omega^{\prime}\right) s \cdot l_{c}} \\
=\hat{a}(\vec{q}, \Omega, 0)+g \int \frac{d \vec{q}^{\prime} d \Omega^{\prime}}{(2 \pi)^{3 / 2}} \bar{\alpha}_{p}\left(\vec{q}+\vec{q}^{\prime}, \Omega+\Omega^{\prime}\right) \\
\quad \times \hat{a}^{\dagger}\left(\vec{q}^{\prime}, \Omega^{\prime}, 0\right) e^{-i \Delta\left(\vec{q}, \Omega, \vec{q}^{\prime}, \Omega^{\prime}\right) \frac{l_{c}}{2}} \\
\quad \times \operatorname{sinc}\left(\frac{\Delta\left(\vec{q}, \Omega, \vec{q}^{\prime}, \Omega^{\prime}\right) l_{c}}{2}\right),
\end{aligned}
$$

where in the last equality we use the identity $\int_{0}^{1} e^{i p s} d s=$ $e^{i p / 2} \operatorname{sinc}(p / 2)$.
We want now the solution for the total field $\hat{A}$, including the linear propagation. Thanks to Eq. (3), we get:

$$
\begin{aligned}
\hat{A}\left(\vec{q}, \Omega, l_{c}\right)= & e^{i k_{s z}(\vec{q}, \Omega) l_{c}}\left[\hat{A}(\vec{q}, \Omega, 0)+g \int \frac{d^{2} \vec{q}^{\prime} d \Omega^{\prime}}{(2 \pi)^{3 / 2}}\right. \\
& \times \bar{\alpha}_{p}\left(\vec{q}+\vec{q}^{\prime}, \Omega+\Omega^{\prime}\right) \hat{A}^{\dagger}\left(\vec{q}^{\prime}, \Omega^{\prime}, 0\right) e^{-i \Delta\left(\vec{q}, \Omega, \vec{q}^{\prime}, \Omega^{\prime}\right) \frac{l_{c}}{2}} \\
& \left.\times \operatorname{sinc}\left(\frac{\Delta\left(\vec{q}, \Omega, \vec{q}^{\prime}, \Omega^{\prime}\right) l_{c}}{2}\right)\right] .
\end{aligned}
$$

\section{APPENDIX B}

In this appendix we show the conditions under which the nearly plane wave pump approximation holds. Our goal is therefore to find when we can substitute $\Delta\left(\vec{q}_{s}, \Omega_{s}, \vec{q}_{p}, \Omega_{p}\right)$, defined in Eq. (12), with $\Delta\left(\vec{q}_{s}, \Omega_{s}, 0,0\right)$ into Eq. (8), i.e., when we can assume:

$$
\begin{array}{r}
e^{i \frac{\Delta\left(\vec{q}_{s}, \Omega_{s}, \vec{q}_{p}, \Omega_{p}\right) l_{c}}{2}} \operatorname{sinc}\left(\frac{\Delta\left(\vec{q}_{s}, \Omega_{s}, \vec{q}_{p}, \Omega_{p}\right) l_{c}}{2}\right) \\
=e^{i \frac{\Delta\left(\vec{q}_{s}, \Omega_{s}, 0,0\right) l_{c}}{2}} \operatorname{sinc}\left(\frac{\Delta\left(\vec{q}_{s}, \Omega_{s}, 0,0\right) l_{c}}{2}\right),
\end{array}
$$

where $\vec{q}_{p}$ and $\Omega_{p}$ lie within the spatiotemporal bandwidth of the pump. We define the function $H(x)=e^{i x} \operatorname{sinc}(x)$ and we write the expansion of $\left.H\left(\Delta\left(\vec{q}_{s}, \Omega_{s}, \vec{q}_{p}, \Omega_{p}\right) l_{c} / 2\right)\right)$ around $\vec{q}_{p}=0$ and $\Omega_{p}=0$. We have:

$$
\begin{aligned}
H( & \left.\frac{\Delta\left(\vec{q}_{s}, \Omega_{s}, \vec{q}_{p}, \Omega_{p}\right) l_{c}}{2}\right) \\
= & H_{0}\left(1+\left.\frac{H_{0}^{\prime}}{H_{0}} \vec{\nabla}_{\vec{q}_{p}} \Delta\left(\vec{q}_{s}, \Omega_{s}, \vec{q}_{p}, \Omega_{p}\right)\right|_{0} \cdot \frac{\vec{q}_{p} l_{c}}{2}\right. \\
& \left.\quad+\left.\frac{H_{0}^{\prime}}{H_{0}} \frac{\partial \Delta\left(\vec{q}_{s}, \Omega_{s}, \vec{q}_{p}, \Omega_{p}\right)}{\partial \Omega_{p}}\right|_{0} \frac{\Omega_{p} l_{c}}{2}\right)+O\left(q_{p}^{2}, \Omega_{p}^{2}\right),
\end{aligned}
$$

where $H_{0}=H\left(\Delta\left(\vec{q}_{s}, \Omega_{s}, 0,0\right) l_{c} / 2\right), \quad H_{0}^{\prime}=H^{\prime}\left(\Delta\left(\vec{q}_{s}, \Omega_{s}\right.\right.$, $\left.0,0) l_{c} / 2\right)$ with $H^{\prime}(x)=d H(x) / d x, \nabla_{\vec{q}_{p}}=\left(\partial / \partial q_{p x}, \partial / \partial q_{p y}\right)$ is the gradient with respect to $\vec{q}_{p}$ and " $\left.\right|_{0}$ " means evaluated at $\vec{q}_{p}=0$ and $\Omega_{p}=0$.

The idea is to find the conditions under which the linear terms in $\vec{q}_{p}$ and $\Omega_{p}$ in Eq. (B2) can be neglected with respect to unity. This will set a condition on the pump waist $\left(w_{p}\right)$ and duration $\left(\tau_{p}\right)$. By assuming, e.g., that the pump has a Gaussian profile both in time and space, we have $q_{p}=2 / w_{p}$ and $\Omega_{p}=$ $2 / \tau_{p}$ (similar relations will hold, as order of magnitude, for any sufficiently smooth pump profile).

Thus

$$
\begin{aligned}
& \left|\frac{H_{0}^{\prime}}{H_{0}} \vec{\nabla}_{\vec{q}_{p}} \Delta\left(\vec{q}_{s}, \Omega_{s}, \vec{q}_{p}, \Omega_{p}\right)\right|_{0} \cdot \frac{\vec{q}_{p} l_{c}}{2} \mid \ll 1 \\
& \quad \Rightarrow w_{p} \gg\left|\frac{H_{0}^{\prime}}{H_{0}} \vec{\nabla}_{\vec{q}_{p}} \Delta\left(\vec{q}_{s}, \Omega_{s}, \vec{q}_{p}, \Omega_{p}\right)\right|_{0} \cdot \frac{\vec{q}_{p}}{q_{p}} \mid l_{c},
\end{aligned}
$$

and

$$
\begin{gathered}
\left|\frac{H_{0}^{\prime}}{H_{0}} \frac{\partial \Delta\left(\vec{q}_{s}, \Omega_{s}, \vec{q}_{p}, \Omega_{p}\right)}{\partial \Omega_{p}}\right| \frac{\Omega_{p} l_{c}}{2} \mid \ll 1 \\
\Rightarrow \tau_{p} \gg\left|\frac{H_{0}^{\prime}}{H_{0}} \frac{\partial \Delta\left(\vec{q}_{s}, \Omega_{s}, \vec{q}_{p}, \Omega_{p}\right)}{\partial \Omega_{p}}\right|_{0} l_{c} .
\end{gathered}
$$


We start from $H_{0}^{\prime} / H_{0}$. Since the function $H_{0}$ assumes its maximum value at $\Delta\left(\vec{q}_{s}, \Omega_{s}, 0,0\right)=\Delta_{\mathrm{pw}}\left(\vec{q}_{s}, \Omega_{s}\right)=0$, i.e., on the phase-matching curve, we calculate the maximum value of the ratio $\left|H^{\prime}(x) / H(x)\right|$ only nearby this region, that is, for $x \in\left[-x_{0}, x_{0}\right]$, where $x_{0}>0$ is defined by the equation $\left|H\left(x_{0}\right)\right|=\operatorname{sinc}\left(x_{0}\right)=0.5$. We find

$$
\max _{x \in\left[-x_{0}, x_{0}\right]}\left|\frac{H^{\prime}(x)}{H(x)}\right|=\left|\frac{H^{\prime}\left(x_{0}\right)}{H\left(x_{0}\right)}\right|=1.32 .
$$

For the constraint on the pump waist (B3) we get:

$$
\begin{aligned}
& \left.\nabla_{\vec{q}_{p}} \Delta\left(\vec{q}_{s}, \Omega_{s}, \vec{q}_{p}, \Omega_{p}\right)\right|_{0} \\
& \quad=\frac{\vec{q}_{s}}{2}\left[\frac{1}{k_{s z}\left(q_{s},-\Omega_{s}\right)}-\frac{1}{k_{s z}\left(q_{s}, \Omega_{s}\right)}\right]+\vec{\rho}_{p},
\end{aligned}
$$

where we remind that $k_{s z}\left(q_{s}, \Omega_{s}\right)=\sqrt{k_{s}^{2}\left(\Omega_{s}\right)-q_{s}^{2}}$, while $\vec{\rho}_{p}=-\left.\nabla_{\vec{q}_{p}} k_{p}\right|_{q_{p}=0, \Omega_{p}=0}$ is the two-dimensional walk-off angle of the extraordinary pump with respect to the $z$ direction. Therefore the constraint (B3) takes the form

$$
\begin{aligned}
w_{p} \gg & 1.32 \mid \frac{\vec{q}_{p}}{q_{p}} \cdot \vec{\rho}_{p} l_{c} \\
& -\frac{\tan \left[\theta_{s}\left(\Omega_{s}\right)\right]+\tan \left[-\theta_{s}\left(-\Omega_{s}\right)\right]}{2} \frac{\vec{q}_{s} \cdot \vec{q}_{p}}{q_{s} q_{p}} l_{c} \mid,
\end{aligned}
$$

where $\tan \left[\theta_{s}\left(\Omega_{s}\right)\right]=q_{s} / k_{s z}\left(q_{s}, \Omega_{s}\right)$ and $\theta_{s}\left(\Omega_{s}\right)$ is the (positive) internal angle formed by the direction of propagation of a signal wave at frequency $\Omega_{s}$, with the $z$ axis. The conjugate wave will then propagate at an angle $-\theta_{s}\left(-\Omega_{s}\right)$, and the second term at right-hand side of Eq. (B7) can be seen as the average lateral displacement of a pair of twin photons when crossing the crystal. Notice that close to degeneracy, this lateral displacement is almost zero because the two photons propagate basically at symmetric directions, while the term becomes important for frequencies well away from degeneracy, where $\theta_{s}\left(\Omega_{s}\right) \neq \theta_{s}\left(-\Omega_{s}\right)$. The first term in Eq. (B7) is the lateral walk-off of the Poynting vector of the extraordinary plane-wave pump with respect to the $z$ axis.

Hence, in general we can conclude that, concerning the pump waist, the NPWPA holds when the pump waist is significantly larger than the lateral walk-off between the the signal and pump waves when crossing the crystal.

For the parameters chosen in our calculations (frequency filter bandwidth $\Delta \Omega_{F}=2 \Omega_{F}=1.37 \times 10^{15} \mathrm{~Hz}, l_{c}=4 \mathrm{~mm}$, $\theta_{p}=33.436^{\circ}$ ), the pump-signal lateral displacement in the direction orthogonal to the pump walk-off $\vec{\rho}_{p}$ (i.e., $\vec{q}_{p} \perp \vec{\rho}_{p}$ ) has a maximum value of:

$$
\left|\frac{\tan \left[\theta_{s}\left(-\Omega_{F}\right)\right]-\tan \left[\theta_{s}\left(\Omega_{F}\right)\right]}{2}\right| l_{c}=58 \mu \mathrm{m},
$$

while in the direction along the pump walk-off $\left(\vec{q}_{p} \| \vec{\rho}_{p}\right)$ it ranges from 240 to $356 \mu \mathrm{m}$, since $\rho_{p} l_{c}=298 \mu \mathrm{m}$.

Regarding the constraint (B4) on the pump duration, we get:

$$
\begin{aligned}
& \left.\frac{\partial \Delta\left(\vec{q}_{s}, \Omega_{s}, \vec{q}_{p}, \Omega_{p}\right)}{\partial \Omega_{p}}\right|_{0} l_{c} \\
& =\frac{l_{c}}{2}\left[\frac{k_{s}\left(\Omega_{s}\right)}{k_{s z}\left(q_{s}, \Omega_{s}\right)} \frac{1}{v_{g s}\left(\Omega_{s}\right)}+\frac{k_{s}\left(-\Omega_{s}\right)}{k_{s z}\left(q_{s},-\Omega_{s}\right)} \frac{1}{v_{g s}\left(-\Omega_{s}\right)}\right] \\
& \quad-\frac{l_{c}}{v_{g p}},
\end{aligned}
$$

where $v_{g s}(\Omega)=\left(d k_{s} / d \Omega\right)^{-1}$ is the signal group velocity, while $v_{g p}=\left.\left(\partial k_{p} / \partial \Omega\right)^{-1}\right|_{q=0, \Omega=0}$ is the group velocity of the pump. If we note now that $l_{c} k_{s}\left(\Omega_{s}\right) / k_{s z}\left(q_{s}, \Omega_{s}\right)=l_{c} \cos \left[\theta_{s}\left(\Omega_{s}\right)\right]$ is the effective distance covered by the signal wave inside the crystal, we can rewrite Eq. (B4) as

$$
\tau_{p} \gg 1.32\left|\frac{\tau_{s}\left(q_{s}, \Omega_{s}\right)+\tau_{s}\left(q_{s},-\Omega_{s}\right)}{2}-\tau_{p}\right|,
$$

where $\tau_{s}\left(q_{s}, \Omega_{s}\right)$ is the crystal crossing time for the signal beam at $\left(q_{s}, \Omega_{s}\right)$, while $\tau_{p}$ is the one for the pump, and we can interpret the term $\left[\tau_{s}\left(q_{s}, \Omega_{s}\right)+\tau_{s}\left(q_{s},-\Omega_{s}\right)\right] / 2$ as the average crystal crossing time for the signal field.

This means that from the temporal point of view, the NPWPA holds if the pump duration is sufficiently greater than the temporal walk-off between the signal and pump beams in crossing the crystal. For the parameters chosen in our calculation we get $\tau_{p} \gg 1.17$ ps.
[1] L.-M. Duan, M. D. Lukin, J. I. Cirac, and P. Zoller, Nature 414, 413 (2001).

[2] S. Lloyd, M. S. Shahriar, J. H. Shapiro, and P. R. Hemmer, Phys. Rev. Lett. 87, 167903 (2001).

[3] S. Du, P. Kolchin, C. Belthangady, G. Y. Yin, and S. E. Harris, Phys. Rev. Lett. 100, 183603 (2008).

[4] J. S. Neergaard-Nielsen, B. M. Nielsen, H. Takahashi, A. I. Vistnes, and E. S. Polzik, Opt. Express 15, 7940 (2007).

[5] A. F. Abouraddy, M. B. Nasr, B. E. A. Saleh, A. V. Sergienko, and M. C. Teich, Phys. Rev. A 65, 053817 (2002).

[6] M. B. Nasr, B. Saleh, A. Sergienko, and M. Teich, Opt. Express 12, 1353 (2004).

[7] V. Giovannetti, S. Lloyd, and L. Maccone, Nature 412, 417 (2001).

[8] V. Giovannetti, S. Lloyd, L. Maccone, and F. N. C. Wong, Phys. Rev. Lett. 87, 117902 (2001).
[9] For a review, see A. Gatti, E. Brambilla, and L. A. Lugiato, in Progress in Optics, edited by E. Wolf (Elsevier B. V., Amsterdam, 2008), Vol. 51, chap. 5, pp. 251.

[10] A. N. Boto, P. Kok, D. S. Abrams, S. L. Braunstein, C. P. Williams, and J. P. Dowling, Phys. Rev. Lett. 85, 2733 (2000).

[11] E. Dauler, G. Jaeger, A. Muller, A. Migdall, and A. Sergienko, J. Res. Natl. Inst. Stand. Technol. 104, 1 (1999).

[12] S. Carrasco, J. P. Torres, L. Torner, A. Sergienko, B. E. A. Saleh, and M. C. Teich, Opt. Lett. 29, 2429 (2004).

[13] S. E. Harris, Phys. Rev. Lett. 98, 063602 (2007).

[14] M. B. Nasr, S. Carrasco, B. E. A. Saleh, A. V. Sergienko, M. C. Teich, J. P. Torres, L. Torner, D. S. Hum, and M. M. Fejer, Phys. Rev. Lett. 100, 183601 (2008).

[15] M. B. Nasr, O. Minaeva, G. N. Goltsman, A. V. Sergienko, B. E. Saleh, and M. C. Teich, Opt. Express 16, 15104 (2008). 
[16] M. B. Nasr, G. D. Giuseppe, B. E. Saleh, A. V. Sergienko, and M. C. Teich, Opt. Commun. 246, 521 (2005).

[17] K. A. O'Donnell and A. B. U'Ren, Opt. Lett. 32, 817 (2007).

[18] S. Carrasco, J. P. Torres, L. Torner, A. Sergienko, B. E. A. Saleh, and M. C. Teich, Phys. Rev. A 70, 043817 (2004).

[19] S. Carrasco, A. V. Sergienko, B. E. A. Saleh, M. C. Teich, J. P. Torres, and L. Torner, Phys. Rev. A 73, 063802 (2006).

[20] S. Carrasco, M. B. Nasr, A. V. Sergienko, B. E. Saleh, M. C. Teich, J. P. Torres, and L. Torner, Opt. Lett. 31, 253 (2006).

[21] M. Hendrych, X. Shi, A. Valencia, and J. P. Torres, Phys. Rev. A 79, 023817 (2009).

[22] A. Gatti, E. Brambilla, L. Caspani, O. Jedrkiewicz, and L. A. Lugiato, Phys. Rev. Lett. 102, 223601 (2009).

[23] E. Brambilla, L. Caspani, L. A. Lugiato, and A. Gatti (2009) (in preparation).

[24] A. Gatti, R. Zambrini, M. San Miguel, and L. A. Lugiato, Phys. Rev. A 68, 053807 (2003).

[25] E. Brambilla, A. Gatti, M. Bache, and L. A. Lugiato, Phys. Rev. A 69, 023802 (2004).

[26] C. K. Hong and L. Mandel, Phys. Rev. A 31, 2409 (1985).

[27] D. N. Klyshko, Photons and Nonlinear Optics (Gordon \& Breach Science, New York, 1988).
[28] J. H. Shapiro and K.-X. Sun, J. Opt. Soc. Am. B 11, 1130 (1994).

[29] R. J. Glauber, Phys. Rev. 130, 2529 (1963).

[30] O. Jedrkiewicz, A. Picozzi, M. Clerici, D. Faccio, and P. Di Trapani, Phys. Rev. Lett. 97, 243903 (2006).

[31] O. Jedrkiewicz, M. Clerici, A. Picozzi, D. Faccio, and P. Di Trapani, Phys. Rev. A 76, 033823 (2007).

[32] M. I. Kolobov and I. V. Sokolov, Zh. Eksp. Teor. Fiz. 96, 1945 (1989) [Sov. Phys. JETP 69, 1097 (1989)].

[33] N. Boeuf, D. Branning, I. Chaperot, E. Dauler, S. Guérin, G. Jaeger, A. Muller, and A. Migdall, Opt. Eng. 39, 1016 (2000).

[34] For a review, see C. Conti, P. Di Trapani, and S. Trillo, in Self-focusing: Past and Present, edited by R. W. Boyd, S. G. Lukishova, and Y. R. Shen (Springer, New York, 2009), Vol. 114 of Topics in Applied physics, chap. 18, pp. 439.

[35] A. Gatti, E. Brambilla, and L. Caspani (in preparation).

[36] O. Jedrkiewicz, Y.-K. Jiang, E. Brambilla, A. Gatti, M. Bache, L. A. Lugiato, and P. Di Trapani, Phys. Rev. Lett. 93, 243601 (2004).

[37] O. Jedrkiewicz, E. Brambilla, M. Bache, A. Gatti, L. A. Lugiato, and P. Di Trapani, J. Mod. Opt. 53, 575 (2006).

[38] Z. Liu and D. Fan, J. Mod. Opt. 45, 17 (1998). 